\title{
Universal Quantum Criticality in the Metal-Insulator Transition of Two-Dimensional Interacting Dirac Electrons
}

\author{
Yuichi Otsuka, ${ }^{1, *}$ Seiji Yunoki, ${ }^{1,2,3}$ and Sandro Sorella ${ }^{1,4,5}$ \\ ${ }^{1}$ Computational Materials Science Research Team, \\ RIKEN Advanced Institute for Computational Science (AICS), \\ Kobe, Hyogo 650-0047, Japan \\ ${ }^{2}$ Computational Condensed Matter Physics Laboratory, RIKEN, Wako, Saitama 351-0198, Japan \\ ${ }^{3}$ Computational Quantum Matter Research Team, RIKEN Center for Emergent Matter Science (CEMS), \\ Wako, Saitama 351-0198, Japan \\ ${ }^{4}$ SISSA-International School for Advanced Studies, Via Bonomea 265, 34136 Trieste, Italy \\ ${ }^{5}$ Democritos Simulation Center CNR-IOM Instituto Officina dei Materiali, \\ Via Bonomea 265, 34136 Trieste, Italy
}

(Received 24 March 2015; revised manuscript received 8 December 2015; published 17 March 2016)

\begin{abstract}
The metal-insulator transition has been a subject of intense research since Mott first proposed that the metallic behavior of interacting electrons could turn to an insulating one as electron correlations increase. Here, we consider electrons with massless Dirac-like dispersion in two spatial dimensions, described by the Hubbard models on two geometrically different lattices, and perform numerically exact calculations on unprecedentedly large systems that, combined with a careful finite-size scaling analysis, allow us to explore the quantum critical behavior in the vicinity of the interaction-driven metal-insulator transition. Thereby, we find that the transition is continuous, and we determine the quantum criticality for the corresponding universality class, which is described in the continuous limit by the Gross-Neveu model, a model extensively studied in quantum field theory. Furthermore, we discuss a fluctuation-driven scenario for the metal-insulator transition in the interacting Dirac electrons: The metal-insulator transition is triggered only by the vanishing of the quasiparticle weight, not by the Dirac Fermi velocity, which instead remains finite near the transition. This important feature cannot be captured by a simple mean-field or Gutzwiller-type approximate picture but is rather consistent with the low-energy behavior of the Gross-Neveu model.
\end{abstract}

DOI: 10.1103/PhysRevX.6.011029

\section{INTRODUCTION}

The metal-insulator transition is one of the most fundamental and yet profound physical phenomenon of quantum mechanics and, in the absence of correlations, is described by the conventional band theory $[1,2]$. A metal is predicted if electrons do not fill an integer number of bands; otherwise, insulating behavior settles because an energy gap is required to excite an electron from a fully occupied to an empty band. With this simple criterion, most insulating and metallic properties were successfully explained [3]. However, it was soon realized by Mott [4] in 1949 that the electron correlations could play a major role in several materials, as they could become insulators even when the band theory predicts metals instead: These are the so-called Mott insulators. Since then, many theoretical and numerical works have

\footnotetext{
*otsukay@ riken.jp
}

Published by the American Physical Society under the terms of the Creative Commons Attribution 3.0 License. Further distribution of this work must maintain attribution to the author(s) and the published article's title, journal citation, and DOI.
Subject Areas: Condensed Matter Physics,

Particles and Fields,

Strongly Correlated Materials tried to shed light on this issue. However, our understanding of interaction-driven metal-insulator transitions still remains rather controversial because strongly correlated systems are hard to solve using both analytical and numerical methods, at least when the spatial dimensionality is larger than 1 [5] but smaller than infinity [6].

In the mid-1960s, Gutzwiller introduced a correlated framework [7] that was later used to derive the properties of the metal-insulator transition as a function of the correlation strength $U$. This framework predicts, for generic lattice models, that for $U$ below the critical point $U_{\mathrm{c}}$, the quasiparticle weight $Z$, which should be exactly 1 in the noninteracting band theory, is strongly renormalized by the correlation and vanishes as $Z \simeq\left(U_{\mathrm{c}}-U\right)$. At the same time, the bandwidth $W$, renormalized by the electron correlations, reduces to zero at the transition in the same way as $Z$ vanishes. The prediction of the Brinkman-Rice approximation [8] was later confirmed and further extended by the dynamical mean-field theory (DMFT) $[9,10]$, an approach that is exact only in the limit of large spatial dimensions.

Here, we focus on a specific realization of the metalinsulator transition in two-dimensional lattice models 
which can be treated with a numerically exact method, i.e., the Hubbard models defined on the honeycomb lattice and on the square lattice with $\pi$ flux penetrating each plaquette. These models are equipped with a free electron energy dispersion with nodal gapless points in the Brillouin zone and with linear dispersion (see Fig. 1), a very peculiar character of the so-called "massless Dirac electrons." We set one electron per site, where the noninteracting band is half-filled and the Fermi surface is constituted by the Dirac points. Because of these gapless Dirac points, it is possible to have a nontrivial metal-insulator transition at a finite value of the correlation strength even in such bipartite lattices [11].
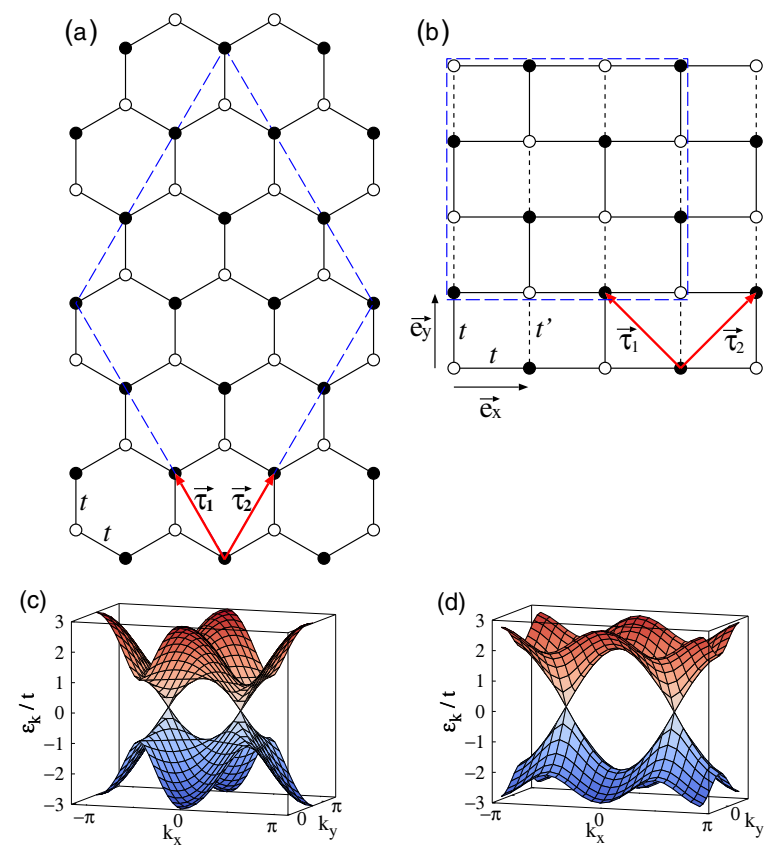

FIG. 1. Lattice structures for (a) the honeycomb lattice model and (b) the $\pi$-flux model, where sites belonging to $A$ and $B$ sublattices are indicated by solid and open circles. The primitive translational vectors are denoted by (a) $\vec{\tau}_{1}=(-\sqrt{3} / 2,3 / 2)$ and $\vec{\tau}_{2}=(\sqrt{3} / 2,3 / 2)$, and (b) $\vec{\tau}_{1}=(-1,1)$ and $\vec{\tau}_{2}=(1,1)$, where the lattice constant between the nearest-neighbor sites is set to 1 . The unit cells for both models thus contain two sites. The nearest-neighbor hopping parameters are indicated by $t$ (solid lines) and $t^{\prime}$ (dotted lines). Note that $t^{\prime}$ is set to be $-t$ for the $\pi$-flux model in (b). The cluster with $L=3(L=4)$ for the honeycomb ( $\pi$-flux) model is indicated by blue dashed line. The unit vector $\vec{e}_{x}\left(\vec{e}_{y}\right)$ along the $x(y)$ direction is also indicated in (b). The noninteracting energy dispersions $\varepsilon_{\mathbf{k}}$ are shown for (c) the honeycomb lattice model and (d) the $\pi$-flux model. The Fermi level is at $\varepsilon_{\mathbf{k}}=0$ for half-filling, and the Dirac points are located exactly at the Fermi level, where the valence and the conduction bands touch with opposite chiralities. Notice that in both models, there are two distinct Dirac points at (c) $\mathbf{k}=(2 \pi / 3)( \pm 1 / \sqrt{3}, 1)$ and (d) $\mathbf{k}=( \pm \pi / 2,0)$, corresponding to two distinct valleys; thus, there are eight components of Dirac fermions in total due to different chiral, spin, and valley degrees of freedom.
Quite recently, a numerical simulation of the Hubbard model on the honeycomb lattice provided evidence for a possible unconventional phase, a spin-liquid phase with no classical order, close to the metal-insulator transition occurring at sufficiently large $U$ [12]. Although there is still activity [13], the possibility of such an intermediate phase between the semimetal (SM) and the antiferromagnetic (AF) Mott insulator now seems rather unlikely, in view of the large-scale simulations that we have reported recently [14], clearly showing that the AF moment develops continuously from zero once we enter the insulating phase. Later studies have also confirmed the simplest scenario of a direct and continuous transition [15-18].

Similarly, a stable spin liquid in interacting Dirac electrons represented by a different model has also been proposed in Ref. [19]. Here, the Hubbard model on the square lattice is studied, in which a flux $\pi$ is added to each plaquette in order to obtain a massless Dirac dispersion in the noninteracting limit [Figs. 1(b) and 1(d)] (referred to as the $\pi$-flux model hereafter). Based on an approximate numerical simulation with relatively small clusters, a spinliquid phase was observed between the $\mathrm{SM}$ and the $\mathrm{AF}$ Mott insulator, which in this case, only has a finite charge gap with a vanishingly small spin gap [19]. This finding is also significant in the context of high- $T_{\mathrm{c}}$ cuprate superconductors because the $\pi$-flux model is considered as one of the relevant models to understand the mechanism of superconductivity [20,21]. However, as in the case of the honeycomb lattice model, this quantum disordered state has also been disputed [17,18,22].

Although it is obviously very important to search for spin-liquid phases in "realistic" models, here we take a different perspective. After several years of effort on these strongly correlated systems, we feel that the time is mature to examine the quantum criticality in the metal-insulator transition of interacting electrons in two spatial dimensions and, in particular, the interacting Dirac electrons described by these two models, where $U_{\mathrm{c}}$ is finite and their groundstate properties can be explored by using an unbiased and formally exact numerical method. This is precisely the main purpose of this paper.

Moreover, it has recently been shown that the interacting Dirac electrons on the honeycomb lattice can be mapped in the continuous limit onto a model well known in quantum field theory, i.e., the Gross-Neveu (GN) model [23] in the chiral Heisenberg universality class with $N=8$ fermion components [24,25]. Since the $\pi$-flux model is expected to have a similar effective theory in the continuous limit, it is reasonable to conjecture that the metal-insulator transition in these two specific lattice models belongs to the same universality class. In order to address this issue, here we perform large-scale quantum Monte Carlo (QMC) calculations and evaluate the critical exponents with a high degree of accuracy. This is indeed made possible because, with the help of the auxiliary field technique [26-28], these 
fermionic models can be studied without the notorious "sign problem" [29,30]. The careful finite-size scaling analysis finds that the critical exponents for these two models are the same within statistical errors and thus confirms the conjecture. Our results represent the first accurate determination of the critical exponents for the GN model in the chiral Heisenberg universality class with $N=8[18,31,32]$.

The other interesting issue to be addressed in this paper is to explore the quantum critical behavior in both metallic and insulating phases in the vicinity of the metal-insulator transition, in particular, the fate of the quasiparticle weight $Z$ and the Fermi velocity $v_{\mathrm{F}}$ when approaching the critical point $U_{\mathrm{c}}$ from the metallic side. For electrons with the usual energy dispersions, such as the one in the square lattice, the Gutzwiller-type approximate description $[7,8]$ and the simple DMFT approach $[9,10]$ predict that $Z$ and $v_{\mathrm{F}}$ are both renormalized by the interaction and vanish at $U_{\mathrm{c}}$. This scenario is valid for any lattice model and in any dimensionality within the Gutzwiller approximation since, within this method, the free electron dispersion is simply renormalized by a Gutzwiller factor $Z$ that vanishes at the transition. Analogously, the same scenario holds within the single-site DMFT [33] because, once the self-energy is assumed to be momentum independent, the free electron dispersion can be renormalized only through the quasiparticle weight $Z$ [9]. Instead, our unbiased and numerically exact calculations support the qualitative prediction based on the renormalization group (RG) analysis for the GN model [34] and the recent numerical results for the honeycomb lattice model obtained by advanced quantum cluster methods [35,36]: By increasing the correlation strength, $Z$ vanishes at the transition, while the Fermi velocity $v_{\mathrm{F}}$ remains finite.

Our large-scale QMC calculations also provide firm numerical evidence for the absence of a spin-liquid phase in between the SM and the AF insulator for the $\pi$-flux model, thus ruling out the possibility of the spin-liquid phase reported previously in Ref. [19]. This is very similar to the case for the honeycomb lattice model, where the originally proposed spin-liquid phase [12] turns out to be rather implausible after our large-scale calculations [14]. The metal-insulator transitions in both models are rather direct and continuous, and can be characterized by the quantum critical behavior of the quasiparticle weight in the metallic phase and the antiferromagnetic order parameter in the insulating phase. These results therefore suggest that the electron correlation alone is not enough, but other factors such as geometrical frustration are required for a magnetically disordered spin-liquid state [37].

The rest of this paper is organized as follows. The definition of the two models and a brief description of the QMC method employed are given in Sec. II. The groundstate phase diagrams are first obtained in Sec. III by a rather conventional way of extrapolating order parameters to the thermodynamic limit. Section IV is devoted to more detailed analysis to determine the critical exponents with high accuracy. The fate of the Fermi velocity is investigated in Sec. V. Finally, the results are discussed in the context of the GN model, followed by an outlook and conclusions, in Sec. VI. The energy-resolved momentum distribution function is described in Appendix A, and the leading correction to the scaling analysis is discussed in Appendix B.

\section{MODELS AND METHOD}

We consider two variants of the Hubbard models in two spatial dimensions, whose low-lying energy states are described by the interacting Dirac fermions with spin- $1 / 2$ degree of freedom at half-filling. The Hamiltonian in standard notation reads

$$
\hat{H}=-\sum_{\langle i, j\rangle} \sum_{s=\uparrow, \downarrow} t_{i j} c_{i s}^{\dagger} c_{j s}+U \sum_{i} n_{i \uparrow} n_{i \downarrow},
$$

where $c_{i s}^{\dagger}$ is the creation operator of the electron at site $i$ and $\operatorname{spin} s(=\uparrow, \downarrow), n_{i s}=c_{i s}^{\dagger} c_{i s}$, and the sum $\langle i, j\rangle$ runs over all pairs of nearest-neighbor sites $i$ and $j$. The first model is defined on the honeycomb lattice with the uniform hopping $t_{i j}=t$ [see Fig. 1(a)]. The second one is on the square lattice with a flux of $\pi$ penetrating each square plaquette, represented, with an appropriate gauge transformation, by $t_{i, i+\vec{e}_{x}}=t$ and $t_{i, i+\vec{e}_{y}}=(-1)^{i_{x}+i_{y}} t$, where the position of site $i$ is given as $i_{x} \vec{e}_{x}+i_{y} \vec{e}_{y}$ and $\vec{e}_{x}\left(\vec{e}_{y}\right)$ denotes the unit vector along the $x(y)$ direction [see Fig. 1(b)].

The clusters considered here consist of $\left(L \vec{\tau}_{1}, L \vec{\tau}_{2}\right)$ with $N_{\mathrm{S}}=2 L^{2}$ sites for the honeycomb lattice model and $\left(L \vec{e}_{x}, L \vec{e}_{y}\right)$ with $N_{\mathrm{s}}=L^{2}$ sites for the $\pi$-flux model, as indicated in Figs. 1(a) and 1(b), respectively, with periodic boundary conditions. The number of electrons is set to be equal to the number of sites in both models. In order to include the Dirac points among the allowed momenta in the noninteracting energy dispersions, $L$ is chosen to be a multiple of three (four) for the honeycomb lattice ( $\pi$-flux) model. The smallest clusters are indicated by dashed lines in Figs. 1(a) and 1(b). The largest clusters considered here are $N_{\mathrm{s}}=2592$ sites for the honeycomb lattice model and $N_{\mathrm{s}}=1600$ for the $\pi$-flux model.

Although the two models are quite different, they are both characterized by the noninteracting energy dispersions $\varepsilon_{\boldsymbol{k}}$ with two gapless Dirac cones, as shown in Figs. 1(c) and $1(\mathrm{~d})$, leading to a semimetallic behavior at half-filling and for small coupling $U / t[29,30]$. The effective low-energy Hamiltonian $\hat{H}_{\text {eff }}^{0}$ in the noninteracting limit at the vicinity of the Dirac points for spin $s$ is described as

$$
\hat{H}_{\mathrm{eff}}^{0}=v_{\mathrm{F}}^{0}\left( \pm \delta k_{x} \sigma_{x}+\delta k_{y} \sigma_{y}\right),
$$

where $\delta \boldsymbol{k}=\left(\delta k_{x}, \delta k_{y}\right)$ is the momentum measured from the Dirac point, $v_{\mathrm{F}}^{0}=3 t / 2(2 t)$ is the Dirac Fermi velocity in 
the noninteracting limit for the honeycomb lattice ( $\pi$-flux) model, and $\vec{\sigma}=\left(\sigma_{x}, \sigma_{y}, \sigma_{z}\right)$ are the Pauli matrices acting on the two different sublattices. As shown below, both models display the metal-insulator transitions at finite critical values $U_{\mathrm{c}} / t$ from the nonmagnetic $\mathrm{SM}$ to the $\mathrm{AF}$ long-range-ordered insulating phase, in good agreement with previous numerical studies $[29,30]$. It should also be noted that in the simplest mean-field picture, the insulating phase emerges because the mass term proportional to $\sigma_{z}$ is introduced in Eq. (2) when an AF order sets in. Therefore, there is no unit-cell doubling in the AF insulating phase for both models [38].

We employ the auxiliary field QMC method [26-28] to investigate the ground-state properties of these two models. The expectation value of a physical observable $\hat{\mathcal{O}}$ over the ground state $\left|\Psi_{0}\right\rangle$ of $\hat{H}$ is obtained by projecting out trial wave functions to the ground state, i.e.,

$$
\langle\hat{\mathcal{O}}\rangle=\left\langle\Psi_{0}|\hat{\mathcal{O}}| \Psi_{0}\right\rangle=\lim _{\tau \rightarrow \infty} O(\tau),
$$

where

$$
O(\tau)=\frac{\left\langle\psi_{\mathrm{L}}\left|e^{-\frac{\tau}{2} \hat{H}} \hat{O} e^{-\frac{\tau}{2} \hat{H}}\right| \psi_{\mathrm{R}}\right\rangle}{\left\langle\psi_{\mathrm{L}}\left|e^{-\tau \hat{H}}\right| \psi_{\mathrm{R}}\right\rangle}
$$

and $\left|\psi_{\mathrm{L}}\right\rangle\left(\left|\psi_{\mathrm{R}}\right\rangle\right)$ is the left (right) trial wave function, chosen to have finite overlap with the exact ground state. We choose $\left|\psi_{\mathrm{L}}\right\rangle$ as a mean-field wave function of Eq. (1) with an $\mathrm{AF}$ order parameter in the $x$ direction, while a Slater determinant of the noninteracting Hamiltonian is used for $\left|\psi_{\mathrm{R}}\right\rangle$, to which a tiny perturbation term is added to remove the degeneracy at the two Dirac points. These choices for the trial wave functions have been shown to yield a particularly fast convergence in the imaginary time projection onto the ground state [14].

The imaginary time evolution operator $e^{-\tau \hat{H}}$ with the projection time $\tau$ is divided into $N_{\tau}$ pieces, i.e., $e^{-\tau \hat{H}}=$ $\left(e^{-\Delta \tau \hat{H}}\right)^{N_{\tau}}$, where $\tau=\Delta \tau N_{\tau}$ and $N_{\tau}$ is the Trotter number (integer). By setting $\Delta \tau t \ll 1$, we can use the Suzuki-Trotter decomposition [39,40], $e^{-\Delta \tau \hat{H}}=$ $e^{-\frac{1}{2} \Delta \tau \hat{H}_{0}} e^{-\Delta \tau \hat{H}_{1}} e^{-\frac{1}{2} \Delta \tau \hat{H}_{0}}+\mathcal{O}\left(\Delta \tau^{3}\right)$, where $\hat{H}_{0}$ is the hopping term and $\hat{H}_{\mathrm{I}}$ is the interacting term of the Hubbard model $\hat{H}$ in Eq. (1). Notice that the systematic error introduced in this decomposition is $\mathcal{O}\left(\Delta \tau^{3}\right)$. The discrete HubbardStratonovich transformation is applied to $e^{-\Delta \tau \hat{H}_{\mathrm{I}}}$, which introduces an auxiliary Ising field at each site, as well as at each imaginary time slice [41]. As shown in Fig. 2, we have confirmed that the systematic errors due to finite $\tau$ and $\Delta \tau$ are sufficiently small, compared to the statistical errors in Monte Carlo importance sampling, when we choose $\tau=L+4$ and $\Delta \tau t=0.1$. More technical details are found in our previous report [14].
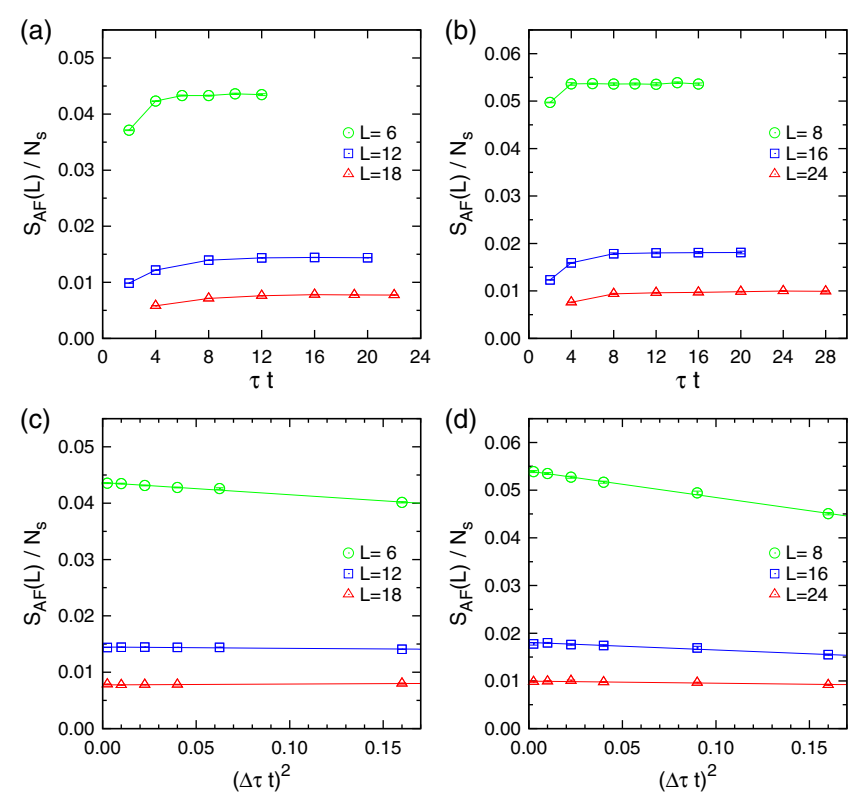

FIG. 2. Upper panels: Convergence of the spin structure factor $S_{\mathrm{AF}}(L)$, defined in Eq. (6), with respect to the projection time $\tau$ for (a) the honeycomb lattice model and (b) the $\pi$-flux model. Lower panels: Extrapolation of $S_{\mathrm{AF}}(L)$ to $\Delta \tau=0$ for (c) the honeycomb lattice model and (d) the $\pi$-flux model. The values shown in (a) and (c) for the honeycomb lattice model are partially taken from Ref. [14]. We set $U / t=4$ (5.8) for the honeycomb lattice ( $\pi$-flux) model. The cluster sizes $L$ used are indicated in the figures. The statistical errors are smaller than the size of the symbols. Straight lines in (c) and (d) are least-square fits to the data with linear functions of $(\Delta \tau t)^{2}$ for different $L$, whereas lines in (a) and (b) are guides to the eye.

\section{GROUND-STATE PHASE DIAGRAM}

In this section, we focus on the continuous nature of the quantum phase transition between the $\mathrm{SM}$ and the $\mathrm{AF}$ insulator. For this purpose, we calculate two fundamental quantities, the staggered magnetization and the quasiparticle weight, which characterize two different aspects across the transition. The former quantity reveals the magnetic transition to the AF state, and the latter one directly captures the metal-insulator transition. These transitions are expected to occur at the same critical $U_{c}$, unless there is an intermediate phase such as the spin-liquid phase [12-16,19]. In this section, we take a conventional and straightforward approach, i.e., by first calculating the staggered magnetization and the quasiparticle weight on different finite clusters and then extrapolating them in the thermodynamic limit, to obtain the ground-state phase diagram as a function of $U / t$.

\section{A. Staggered magnetization}

The staggered magnetization on each finite cluster with a linear dimension $L$, expressed as $m_{\mathrm{s}}(L)$, is calculated from the spin structure factor $S_{\mathrm{AF}}(L)$, i.e., 


$$
m_{\mathrm{s}}(L)=\sqrt{\frac{S_{\mathrm{AF}}(L)}{N_{\mathrm{s}}}},
$$

where

$$
S_{\mathrm{AF}}(L)=\frac{1}{N_{\mathrm{s}}}\left\langle\left(\sum_{i \in A} \vec{S}_{i}-\sum_{i \in B} \vec{S}_{i}\right)^{2}\right\rangle,
$$

$\vec{S}_{i}=\frac{1}{2} \sum_{s, s^{\prime}} c_{i s}^{\dagger}(\vec{\sigma})_{s s^{\prime}} c_{i s^{\prime}}$ is the spin operator at site $i$, and the sum $i \in A(B)$ in Eq. (6) runs over sites belonging to $A(B)$ sublattices [see Figs. 1(a) and 1(b)]. The AF order parameter $m_{\mathrm{s}}$ is determined by extrapolating $m_{\mathrm{s}}(L)$ in the thermodynamic limit, i.e.,

$$
m_{\mathrm{s}}=\lim _{L \rightarrow \infty} m_{\mathrm{s}}(L)
$$

At a fixed value of $U / t$, the staggered magnetizations $m_{\mathrm{s}}(L)$ are calculated on clusters with $L=6,9,12,15,18$, 24 , and 36 for the honeycomb lattice model, and with $L=8,12,15,18,24,32$, and 40 for the $\pi$-flux model, and are extrapolated to the thermodynamic limit using polynomial functions in $1 / L$. The typical results are shown in Fig. 3. It is observed that such a simple functional form represents the data rather well, and thus we can estimate the $\mathrm{AF}$ order parameter $m_{\mathrm{s}}$ reasonably accurately. The results for the extrapolated AF order parameter $m_{\mathrm{s}}$ are summarized in Fig. 4.

The critical points $U_{\mathrm{c}}$, above which the $\mathrm{AF}$ order parameter $m_{\mathrm{s}}$ is finite, and the critical exponents $\beta$ are estimated by assuming a form of the AF order parameter as a function of $U$ as

$$
m_{\mathrm{s}} \sim\left(U-U_{\mathrm{c}}\right)^{\beta} .
$$

The estimated $U_{\mathrm{c}} / t$ is $3.85 \pm 0.02$ for the honeycomb lattice model and $5.65 \pm 0.05$ for the $\pi$-flux model, as
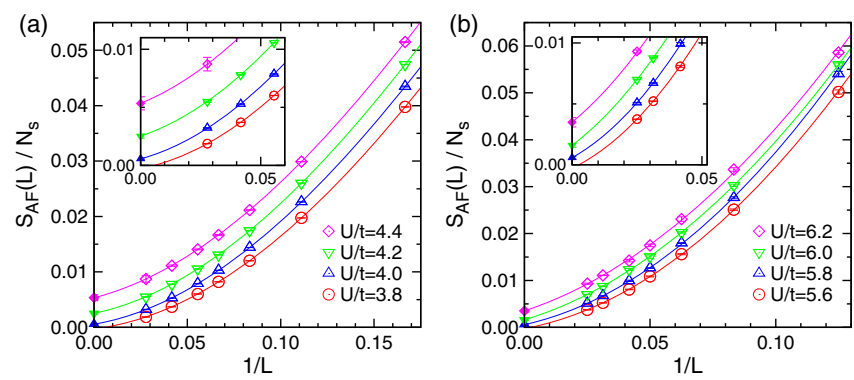

FIG. 3. Extrapolation of the spin structure factor $S_{\mathrm{AF}}(L)$ to the thermodynamic limit for (a) the honeycomb lattice model and (b) the $\pi$-flux model with different $U / t$ values indicated in the figures. Solid curves are least-square fits of the data by cubic (quadratic) polynomials in $1 / L$ for the honeycomb lattice ( $\pi$-flux) model. Insets show enlarged plots for large $L$. The extrapolated values to the thermodynamic limit are also indicated at $1 / L=0$.
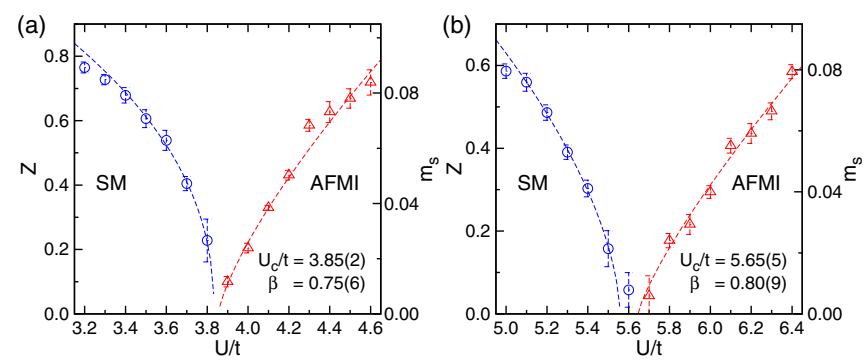

FIG. 4. The ground-state phase diagrams for (a) the honeycomb lattice model and (b) the $\pi$-flux model. Open triangles and open circles represent the $\mathrm{AF}$ order parameter $m_{\mathrm{s}}$ and the quasiparticle weight $Z$, respectively. The critical $U_{\mathrm{c}}$ estimated from $m_{\mathrm{s}}$, assuming a form of $m_{\mathrm{s}} \sim\left(U-U_{\mathrm{c}}\right)^{\beta}$ (red dashed lines), is indicated in the figures along with the critical exponent $\beta$. The chi-square values divided by degrees of freedom for this estimation are 0.88 for (a) and 1.05 for (b). Blue dashed line for $Z$ is a guide to the eye. SM and AFMI stand for semimetal and antiferromagnetic insulator, respectively. Most of values for $m_{\mathrm{s}}$ shown in (a) for the honeycomb lattice model are taken from Ref. [14].

indicated in Fig. 4. Notice that $U_{\mathrm{c}}$ for the $\pi$-flux model is larger than that for the honeycomb lattice model. This is easily understood because $v_{\mathrm{F}}^{0}$ for the former model is larger than that for the latter model [see Eq. (2) and also Figs. 1(c) and 1(d)], and thus a larger $U$ is required to induce the $\mathrm{AF}$ order. Although $U_{\mathrm{c}} / t$ is different for these two models, our calculations find that the critical exponents $\beta$ for the two models are the same within statistical errors, i.e., $\beta=0.75 \pm 0.06$ for the honeycomb lattice model and $0.80 \pm 0.09$ for the $\pi$-flux model, as indicated in Fig. 4, which will be confirmed in more detail by a careful and accurate finite-size scaling analysis in Sec. IV.

\section{B. Quasiparticle weight}

Next, we study the metal-insulator transition by considering the momentum distribution function, i.e., the ground-state occupation of the one-electron states labeled by momentum $\boldsymbol{k}$, spin $s$, and noninteracting energy $\varepsilon_{\boldsymbol{k}}$. We calculate this occupation number $n\left(\varepsilon_{k}\right)$ [defined in Eq. (A3)] as a function of $\varepsilon_{k}$, where we average over equivalent momenta with the same energy (see Appendix A for details). Typical results of the "energy-resolved" momentum distribution function $n\left(\varepsilon_{k}\right)$ are shown in Fig. 5 for both models. For $U<U_{\mathrm{c}}$, a jump in the momentum distribution function $n\left(\varepsilon_{k}\right)$ occurs for $\varepsilon_{k} \rightarrow 0$ when $\boldsymbol{k}$ approaches the Dirac points. The singularity in $n\left(\varepsilon_{k}\right)$ implies a long-distance power-law behavior in the density matrix $\left\langle c_{i s}^{\dagger} c_{j s}\right\rangle$ in real space, which is the fingerprint of a metal. From general grounds [42], by applying the well-known "Migdal theorem" [43], the quasiparticle weight $Z$ can be related to the jump in the momentum distribution function. Therefore, we can have direct access to $Z$ in the thermodynamic limit. However, the finite-size 

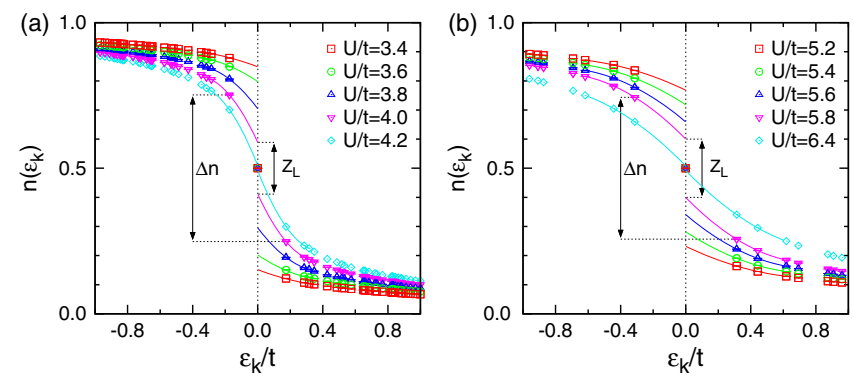

FIG. 5. Energy-resolved momentum distribution function $n\left(\varepsilon_{\boldsymbol{k}}\right)$ near the Fermi level, indicated by a vertical dotted line at $\varepsilon_{k}=0$, for (a) the honeycomb lattice model $(L=36)$ and (b) the $\pi$-flux model $(L=40)$ with different values of $U / t$ indicated in the figures. Solid curves are least-square fits of the three data points closest to the Fermi level with $\varepsilon_{\boldsymbol{k}}<0$ and $\varepsilon_{\boldsymbol{k}}>0$ using quadratic polynomials of $\varepsilon_{k}$. Notice that, because of the particle-hole symmetry, $n\left(\varepsilon_{\boldsymbol{k}}>0\right)=1-n\left(\varepsilon_{\boldsymbol{k}}<0\right)$, and thus $n\left(\varepsilon_{\boldsymbol{k}}=0\right)=1 / 2$. $Z_{L}$ and $\Delta n(u, L)$ are indicated in (a) for $U / t=4$ and in (b) for $U / t=5.8$.

effects are rather significant and need to be carefully controlled in order to reach definite conclusions based on the available finite-size calculations.

It should also be noticed in Fig. 5 that the energyresolved momentum distribution function $n\left(\varepsilon_{k}\right)$ becomes smooth without a visible discontinuity at the Fermi level for large $U / t$. This is interpreted as an exponential decay of the density matrix at large distances because the density matrix is simply the Fourier transform of the momentum distribution function. This clearly indicates the presence of a gap in the charge sector.

We find that the following procedure works for estimating $Z$ in the thermodynamic limit. Since the energyresolved momentum distribution function $n\left(\varepsilon_{k}\right)$ is smooth near $\varepsilon_{\boldsymbol{k}}=0$, we analyze $n\left(\varepsilon_{\boldsymbol{k}}\right)$ calculated on different finite clusters by extrapolating it to $\varepsilon_{k}=0$ with a polynomial function. More specifically, the quasiparticle weight on each finite cluster, $Z_{L}$, is defined as the jump in $n\left(\varepsilon_{k}\right)$, which is evaluated by extrapolating to the Fermi level $\left(\varepsilon_{k}=0\right)$ the closest three data points of $n\left(\varepsilon_{k}\right)$ for $\varepsilon_{k}<0$ or $\varepsilon_{k}>0$ with a quadratic polynomial, as shown in Fig. 5. Notice that because of the particle-hole symmetry, the two extrapolations using the data points for $\varepsilon_{\boldsymbol{k}}<0$ and for $\varepsilon_{k}>0$ are related, and in practice, only one of the two is necessary.

The extrapolated quantity $Z_{L}$ for finite $L$ is certainly much closer to the value in the thermodynamic limit and quite generally converges smoothly to the quasiparticle weight

$$
Z=\lim _{L \rightarrow \infty} Z_{L}
$$

with a quadratic polynomial function of $1 / L$, as shown in Fig. 6. However, this extrapolation is valid only in the metallic regime, where the assumed polynomial
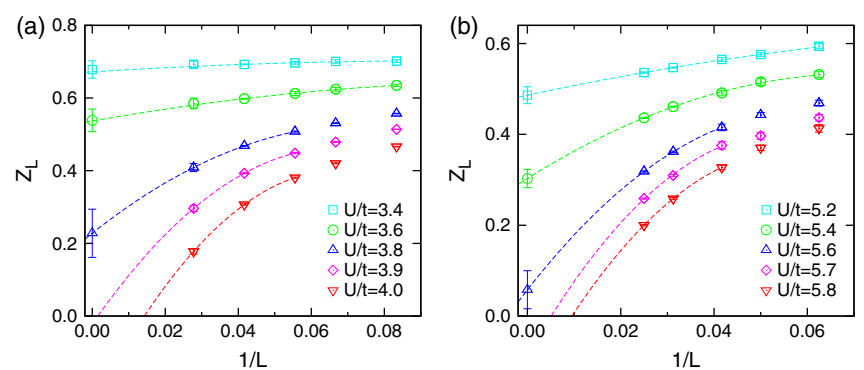

FIG. 6. Extrapolation of the quasiparticle weight $Z_{L}$ to the thermodynamic limit for (a) the honeycomb lattice model and (b) the $\pi$-flux model with different values of $U / t$ indicated in the figures. Dashed curves are least-square fits for the largest sizes with quadratic polynomials of $1 / L$, which are extrapolated to the thermodynamic limit to estimate the quasiparticle weight $Z$ indicated at $1 / L=0$.

convergence in $1 / L$ is justified. Indeed, we find in Fig. 6 that, for larger $U / t, Z_{L}$ is extrapolated to a negative value, instead of being positive. This is clearly an inconsistent extrapolation because $Z_{L}$ in the insulating phase is expected to converge exponentially. Nevertheless, these inconsistent extrapolations are very useful as they allow us to identify the extension of the metallic region and determine the critical $U_{\mathrm{c}}^{\mathrm{MI}}$ for the metal-insulator transition. It is indeed estimated in Fig. 6 that $U_{\mathrm{c}}^{\mathrm{MI}} / t \sim 3.9$ for the honeycomb lattice model and $U_{\mathrm{c}}^{\mathrm{MI}} / t \sim 5.6$ for the $\pi$-flux model, which are in excellent agreement with the critical $U_{\mathrm{c}}$ for the AF order (see Fig. 4).

The results for the quasiparticle weight $Z$ in the thermodynamic limit as a function of $U / t$ are summarized in Fig. 4 for both models. The obtained $Z$ for $U<U_{\mathrm{c}}^{\mathrm{MI}}$ appears well behaved and can provide the critical $U_{\mathrm{c}}^{\mathrm{MI}}$ by assuming that

$$
Z \sim\left(U_{\mathrm{c}}^{\mathrm{MI}}-U\right)^{\eta_{Z}}
$$

for $U<U_{\mathrm{c}}^{\mathrm{MI}}$ close to $U_{\mathrm{c}}^{\mathrm{MI}}$. We find that $U_{\mathrm{c}}^{\mathrm{MI}} / t=3.83 \pm 0.05$ for the honeycomb lattice and $U_{\mathrm{c}}^{\mathrm{MI}} / t=5.56 \pm 0.06$ for the $\pi$-flux model [44]. The critical values for $U_{\mathrm{c}}^{\mathrm{MI}}$ are thus consistent with those for $U_{\mathrm{c}}$ determined from the $\mathrm{AF}$ order parameter $m_{\mathrm{s}}$ within the statistical errors. These results clearly imply that the transition from the SM to the AF insulator is continuous in both models, where the insulating behavior shows up immediately when the AF order is developed for $U \geq U_{\mathrm{c}}$, i.e., $U_{\mathrm{c}}^{\mathrm{MI}}=U_{\mathrm{c}}$. Therefore, our large-scale calculations exclude the intermediate phases previously reported in Refs. [12] and [19] and reveal a continuous transition between the SM and the AF insulator. As shown in Sec. IV, the careful finite-size scaling analysis finds that the data-collapse fits for both $m_{\mathrm{s}}$ and $Z$ are convincing by setting $U_{\mathrm{c}}^{\mathrm{MI}}=U_{\mathrm{c}}$, also suggesting that the quantum critical points for these two quantities are located at the same $U$ value. 
Finally, we remark on a semantic issue. The "Mott transition" is very widely used to describe a metalinsulator transition driven by the electron correlation, regardless of whether the symmetry may or may not be broken in the insulating phase. There is certainly more confusion and ambiguity on how to properly define a "Mott insulator," especially in our case when the unit cell contains an even number of electrons and the AF order found in the insulating phase does not break the translation symmetry. However, this semantic issue is irrelevant to the main purpose of our study and does not change our conclusions.

\section{FINITE-SIZE SCALING ANALYSIS}

Having established the continuous character of the transition, let us now evaluate the critical exponents that characterize the quantum phase transition. For this purpose, here we employ the careful finite-size scaling analysis for staggered magnetization and quasiparticle weight.

\section{A. Staggered magnetization}

For the staggered magnetization, we make use of the standard finite-size scaling ansatz [45-47],

$$
m_{\mathrm{s}}(u, L)=L^{-\beta / \nu}\left(1+c L^{-\omega}\right) f_{m}\left(u L^{1 / \nu}\right),
$$

where $\nu$ is the critical exponent of the correlation length $\xi \simeq\left|U-U_{\mathrm{c}}\right|^{-\nu}, f_{m}\left(u L^{1 / \nu}\right)$ denotes a model-dependent scaling function, and $u=\left(U-U_{\mathrm{c}}\right) / U_{\mathrm{c}}$ is the reduced coupling. Notice that the $u$ dependence of $m_{\mathrm{s}}(L)$ in Eq. (5) is explicitly indicated in Eq. (11) as $m_{\mathrm{s}}(u, L)$. In the above finite-size scaling ansatz, we also take into account the leading correction term, a term proportional to $c L^{-\omega}$, with $c$ and $\omega$ being additional fitting parameters [48], which is, however, expected to be less important for sufficiently large cluster sizes. The finite-size scaling analysis is performed with a recently proposed method based on the Bayesian statistics [49]. The remarkable advantages of this method are (i) the weak dependence on the initial fitting parameters and (ii) the applicability to a wide range of the reduced coupling $u$.

In order to estimate reliable error bars for the fitting parameters in Eq. (11), we adopt a straightforward resampling technique. The fitting procedure is summarized as follows. First, we prepare a data set to be fitted, based on the raw QMC data of $m_{\mathrm{s}}(u, L)$ for various $u$ and $L$, by adding to $m_{\mathrm{s}}(u, L)$ a Gaussian-distributed noise with the zero average and the standard deviation estimated by the QMC calculations for $m_{\mathrm{s}}(u, L)$. Second, we pick up at random initial values of the fitting parameters $U_{\mathrm{c}} / t, \nu, \beta$, and $\omega$ around the optimal ones, namely, $U_{\mathrm{c}} / t=3.8$ (5.5) for the honeycomb lattice ( $\pi$-flux) model, and $\nu=1.0, \beta=0.8$, and $\omega=0.8$ for both models. Third, with these initial fitting parameters, we perform the data-collapse analysis for all the resampled data for $m_{\mathrm{s}}(u, L)$ based on the Bayesian statistics [49] and obtain the best converged values of $U_{\mathrm{c}} / t, \nu, \beta$, and $\omega$. We repeat this procedure typically a few thousand times to average the converged parameters and estimate the statistical errors. Typical examples of the resampling procedure are shown in Figs. 7 and 8. The clear advantage of the resampling technique is that the degree of uncertainty of the fitting parameters can be immediately verified, and therefore, a reliable estimate of the error bars can be safely obtained.

The results of the data collapse for the staggered magnetization $m_{\mathrm{s}}(u, L)$ are shown in Fig. 9, confirming that our numerical calculations are quite accurate for this quantity since the data for different $L$ collapse almost perfectly into a universal curve. We find that the critical exponents are quite stable and that they converge to $\nu \simeq 1$ and $\beta \simeq 0.75$ for both models, as indicated in Fig. 9. It should be noticed that, for both models, the values of $U_{\mathrm{c}}$, obtained from the data-collapse plots in Fig. 9, agree within 2 standard deviations with the ones estimated
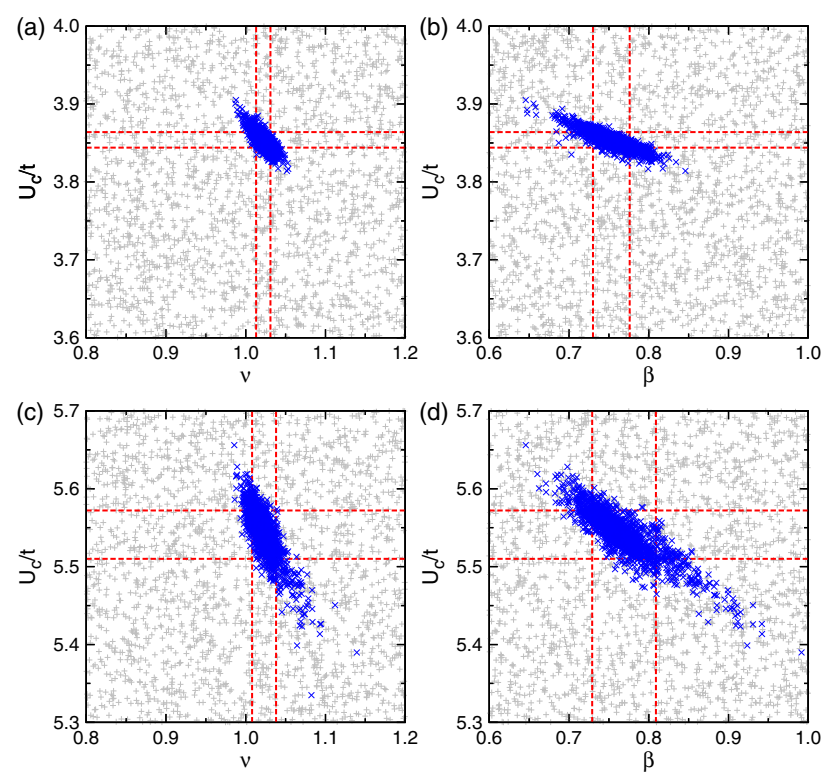

FIG. 7. Scattering plots of the computed fitting parameters (blue crosses) for $m_{\mathrm{s}}(u, L)$ obtained with the Bayesian method [49] and the resampling procedure described in the text. Here, the data-collapse fits for $m_{\mathrm{s}}(u, L)$ with the leading correction term given in Eq. (11) are performed several thousand times: We generate different input data sets (i.e., resampled data sets) that are statistically consistent with the raw QMC data of $m_{\mathrm{s}}(u, L)$, and we employ the Bayesian method to perform the data collapse fit for each resampled data set with a different set of initial fitting parameters (gray symbols) prepared randomly. We use $L \geq 15$ for the honeycomb lattice model [(a) and (b)] and $L \geq 20$ for the $\pi$-flux model [(c) and (d)]. The vertical and horizontal dashed lines indicate 1 standard deviation from the averaged values of the computed fitting parameters. 

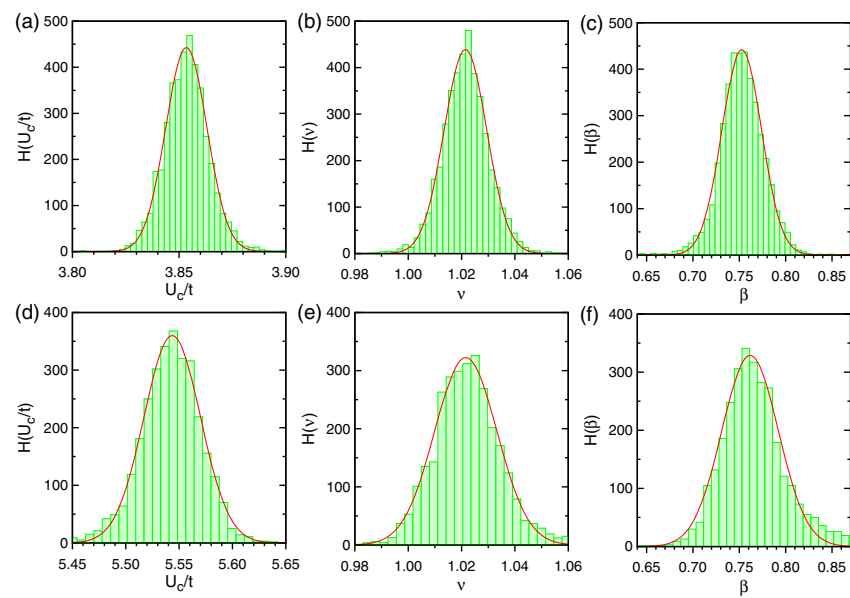

FIG. 8. Histograms of the computed fitting parameters for $m_{\mathrm{s}}(u, L)$ obtained with the Bayesian method [49] and the resampling procedure described in the text. The same data sets as in Fig. 7 are used for (a)-(c) the honeycomb lattice model and for (d)-(f) the $\pi$-flux model. Solid curves are fits to the histograms with a Gaussian function. These results clearly show that, after performing the Bayesian analysis several thousand times for different resampled data sets generated statistically consistent with the raw QMC data, the computed fitting parameters are distributed normally, and therefore, we can reasonably estimate their average values and the corresponding statistical errors.
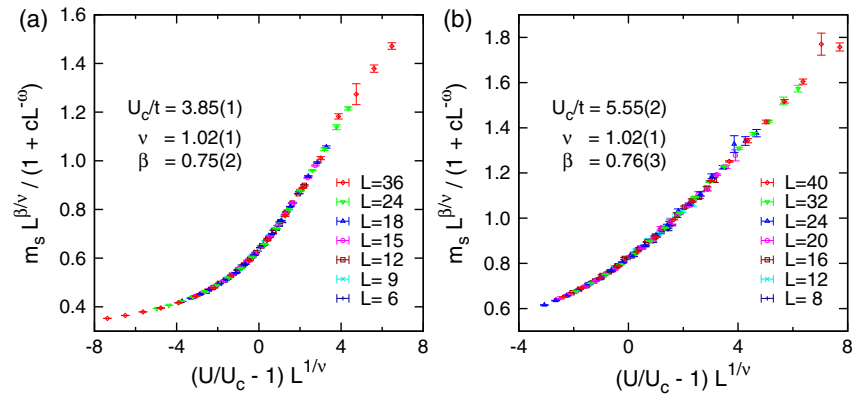

FIG. 9. Data-collapse fits of the staggered magnetization $m_{\mathrm{s}}$ for (a) the honeycomb lattice model and (b) the $\pi$-flux model. The scaling form is given in Eq. (11), where the fitting parameters $U_{\mathrm{c}}$, $\nu, \beta, c$, and $\omega$ are determined by the resampling technique using the data sets for $L \geq 15$ in (a) and $L \geq 20$ in (b) (see also Table III in Appendix B). The obtained values $U_{\mathrm{c}}, \nu$, and $\beta$ as well as the system sizes $L$ used are indicated in the figures. The number in parentheses denotes the estimated error in the last digit.

straightforwardly by extrapolating $m_{\mathrm{s}}(L)$ to the thermodynamic limit for each $U$ (see Figs. 3 and 4).

It should be noted here that very recently a similar QMC method has been applied to the honeycomb lattice model and the $\pi$-flux model by Parisen Toldin et al. in Ref. [18]. In their report, the critical exponents for the honeycomb lattice model are estimated as $\nu \simeq 0.84$ and $\beta \simeq 0.71$, and are claimed to be consistent with those
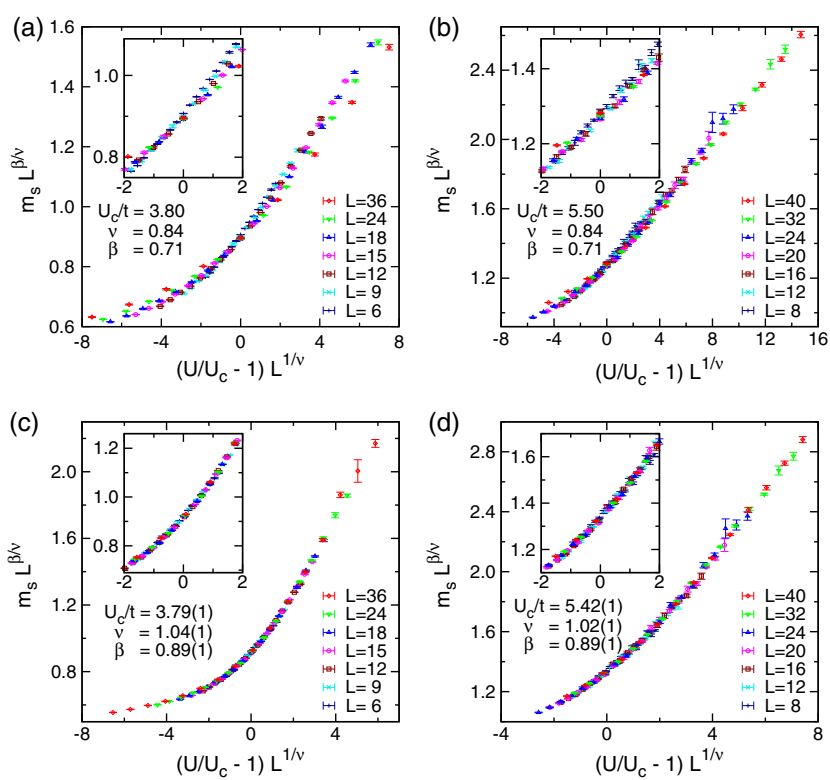

FIG. 10. Data-collapse plots of the staggered magnetization $m_{\mathrm{s}}$ for (a) the honeycomb lattice model and (b) the $\pi$-flux model with fixed values of $U_{c}, \nu$, and $\beta$, taken from Ref. [18]. In Ref. [18], the critical exponents $\nu$ and $\beta$ are estimated, based on their numerical data and renormalization group analysis, only for the honeycomb lattice model, and they are assumed to be the same for the $\pi$-flux model. For comparison, data-collapse fits are also shown in the lower panels for (c) the honeycomb lattice model and (d) the $\pi$-flux model, where $U_{\mathrm{c}}, \nu$, and $\beta$ are determined by the resampling technique using the data sets for $L \geq 9$ in (c) and $L \geq 12$ in (d) (see also Table II in Appendix B). In all figures, the same data are used as those in Fig. 9, but the leading correction term is not considered, i.e., $c=0$ in Eq. (11), for a fair comparison.

obtained by the $\epsilon$ expansion for the GN model [25] (see also Table I), while the critical exponents for the $\pi$-flux model are unavailable. We argue that the disagreement of the critical exponents between their estimations and ours for the honeycomb model and the difficulty to determine the critical exponents for the $\pi$-flux model in Ref. [18] are due to the limited lattice sizes used in Ref. [18], i.e., up to 648 sites for the honeycomb lattice model and 784 sites for the $\pi$-flux model. As shown in Figs. 10(a) and 10(b), our numerical data do not provide perfect data-collapse plots for both models when we fix $U_{\mathrm{c}}, \nu$, and $\beta$, which are reported in Ref. [18]. However, the scattering of the data is particularly evident only for the two largest sizes, not studied in Ref. [18]. Indeed, if these largest data sets are excluded from the plots, the data collapse seems acceptable even when using the values of $U_{\mathrm{c}}, \nu$, and $\beta$ reported in Ref. [18], especially for the honeycomb lattice model shown in Fig. 10(a). On the other hand, Figs. 10(c) and 10(d) confirm that our estimation of the critical values even without the leading correction term (see Appendix B for the details) yields an excellent data collapse. 
TABLE I. Critical exponents $\nu, \beta$, and $\eta_{\psi}$ of the interaction-driven phase transition in interacting Dirac fermions in $d=2+1$ for the lattice models (honeycomb lattice and $\pi$-flux models) and the effective continuous models (Gross-Neveu models) with the total number $N$ of fermion components. Different classes correspond to different symmetries broken in the ordered phases. Numbers in parentheses for $\nu, \beta$, and $\eta_{\psi}$ indicate statistical errors in the last digits. For comparison, the critical exponents for other related models with $N=4$ and 8 , belonging to different universality classes, are also listed. FRG stands for the functional renormalization group method.

\begin{tabular}{|c|c|c|c|c|c|c|}
\hline Model & $N$ & Class & Method & $\nu$ & $\beta$ & $\eta_{\psi}$ \\
\hline Honeycomb & 8 & Chiral Heisenberg & Monte Carlo (present) & $1.02(1)$ & $0.76(2)$ & $0.20(2)$ \\
\hline$\pi$-flux & 8 & Chiral Heisenberg & Monte Carlo (present) & $1.02(1)$ & $0.74(3)$ & $0.23(2)$ \\
\hline Honeycomb & 8 & Chiral Heisenberg & Monte Carlo [18] & $0.84(4)$ & $0.71(8)$ & $\cdots$ \\
\hline Gross-Neveu & 8 & Chiral Heisenberg & $4-\epsilon$, first order $[31,50]$ & 0.851 & 0.824 & 0.167 \\
\hline Gross-Neveu & 8 & Chiral Heisenberg & $4-\epsilon$, second order $[31,50]$ & 1.01 & 0.995 & 0.101 \\
\hline Gross-Neveu & 8 & Chiral Heisenberg & FRG [32] & 1.31 & 1.32 & 0.08 \\
\hline Gross-Neveu & 8 & Chiral Heisenberg & $4-\epsilon$, first order $[25,50]$ & 0.882 & 0.794 & 0.3 \\
\hline Gross-Neveu & 4 & Chiral Heisenberg & $4-\epsilon$, first order [31] & 0.882 & 0.794 & 0.3 \\
\hline Gross-Neveu & 4 & Chiral Heisenberg & $4-\epsilon$, second order $[31]$ & 1.083 & 1.035 & 0.242 \\
\hline Gross-Neveu & 8 & Chiral Ising & Monte Carlo [51] & $1.00(4)$ & $0.88(4)$ & $\cdots$ \\
\hline Gross-Neveu & 8 & Chiral Ising & Monte Carlo [52] & $0.83(1)$ & $0.67(1)$ & $0.38(1)$ \\
\hline Gross-Neveu & 8 & Chiral Ising & $4-\epsilon$, first order $[31]$ & 0.738 & 0.631 & 0.071 \\
\hline Gross-Neveu & 8 & Chiral Ising & $4-\epsilon$, second order [31] & 0.850 & 0.722 & 0.065 \\
\hline Gross-Neveu & 8 & Chiral Ising & $2+\epsilon$, third order [53] & 1.309 & 1.048 & 0.081 \\
\hline Gross-Neveu & 8 & Chiral Ising & $O\left(1 / N^{2}\right)[51,54,55]$ & 0.829 & 0.723 & 0.044 \\
\hline Gross-Neveu & 8 & Chiral Ising & FRG $[56,57]$ & 1.018 & 0.894 & 0.032 \\
\hline Gross-Neveu & 8 & Chiral Ising & FRG [32] & 1.018 & 0.896 & 0.032 \\
\hline Honeycomb & 4 & Chiral Ising & Monte Carlo [58] & $0.80(3)$ & $0.52(2)$ & $\cdots$ \\
\hline$\pi$-flux & 4 & Chiral Ising & Monte Carlo [58] & $0.80(3)$ & $0.53(4)$ & $\cdots$ \\
\hline Honeycomb & 4 & Chiral Ising & Monte Carlo [59] & $0.77(3)$ & $0.60(3)$ & $\ldots$ \\
\hline$\pi$-flux & 4 & Chiral Ising & Monte Carlo [59] & $0.79(4)$ & $0.67(4)$ & $\cdots$ \\
\hline Honeycomb & 4 & Chiral Ising & Monte Carlo [60] & $0.74(4)$ & $0.47(4)$ & $\cdots$ \\
\hline Gross-Neveu & 4 & Chiral Ising & $4-\epsilon$, first order $[31]$ & 0.709 & 0.559 & 0.100 \\
\hline Gross-Neveu & 4 & Chiral Ising & $4-\epsilon$, second order [31] & 0.797 & 0.610 & 0.110 \\
\hline Gross-Neveu & 4 & Chiral Ising & FRG [56,57] & 0.927 & 0.707 & 0.071 \\
\hline Gross-Neveu & 8 & Chiral $X Y$ & $4-\epsilon$, first order [31] & 0.726 & 0.619 & 0.071 \\
\hline Gross-Neveu & 8 & Chiral $X Y$ & $4-\epsilon$, second order [31] & 0.837 & 0.705 & 0.063 \\
\hline Gross-Neveu & 4 & Chiral $X Y$ & $4-\epsilon$, first order [31] & 0.7 & 0.55 & 0.1 \\
\hline Gross-Neveu & 4 & Chiral $X Y$ & $4-\epsilon$, second order [31] & 0.799 & 0.607 & 0.106 \\
\hline
\end{tabular}

\section{B. Quasiparticle weight}

As far as the critical behavior in the charge sector is concerned, the scaling ansatz is applied to the jump $\Delta n(u, L)$ of the momentum distribution function $n\left(\varepsilon_{k}\right)$ across the Fermi level with a form

$$
\Delta n(u, L)=L^{-\eta_{\psi}} f_{n}\left(u L^{1 / \nu}\right)
$$

where $\eta_{\psi}$ is the anomalous dimension of the fermion field $\Psi$ [61] and $f_{n}\left(u L^{1 / \nu}\right)$ is a scaling function. The jump $\Delta n(u, L)$ is simply obtained as a difference of $n\left(\varepsilon_{\boldsymbol{k}}\right)$ at the two closest points to the Fermi level, i.e., the ones above and below the Fermi level (see Fig. 5). Here, we do not use $Z_{L}$ as the scaling quantity in Eq. (12) to avoid possible artifacts caused by the extrapolation procedure for $Z_{L}$, where $n\left(\varepsilon_{\boldsymbol{k}}\right)$ is extrapolated to $\varepsilon_{\boldsymbol{k}}=0$. The finite-size scaling for $\Delta n(u, L)$ is certainly more difficult because
$\Delta n(u, L)$ is the direct finite-size jump of $n\left(\varepsilon_{\boldsymbol{k}}\right)$ across the Fermi level and is much larger than the quasiparticle weight $Z$ in the thermodynamic limit, as shown in Fig. 5. Therefore, we fix the critical $U_{\mathrm{c}}$ and the exponent $\nu$ in Eq. (12) to the values already determined by the finite-size scaling analysis on $m_{\mathrm{s}}(u, L)$. Moreover, we do not consider the correction term in the finite-size scaling analysis (see Appendix B for the case with the leading correction term). The remaining parameter $\eta_{\psi}$ is determined using the resampling technique described in Sec. IVA.

Despite the above simplifications, we find in Fig. 11 that the collapse plots are also excellent in this case, suggesting that the lattice sizes considered here are large enough and that we can also faithfully describe the critical behavior in the charge sector. The values obtained for the critical exponent $\eta_{\psi}$ are found to be the same for both models $\left(\eta_{\psi} \simeq 0.21-0.22\right)$ within the statistical errors. However, these values differ from those obtained 

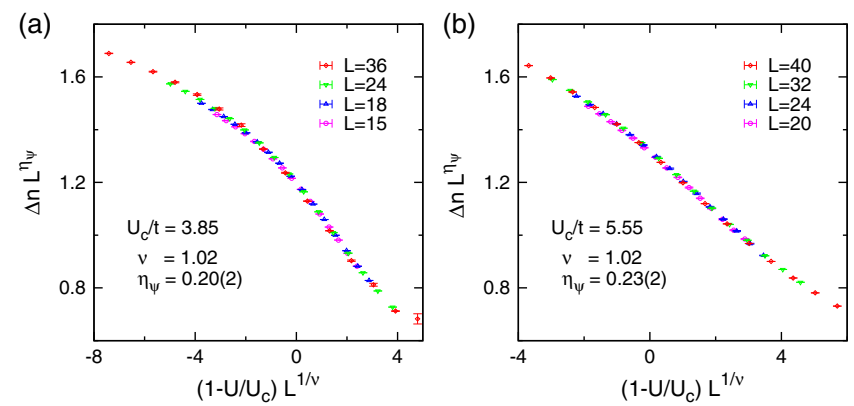

FIG. 11. Data-collapse fits of $\Delta n(u, L)$ for (a) the honeycomb lattice model and (d) the $\pi$-flux model. The scaling form is given in Eq. (11), where $U_{\mathrm{c}}$ and $\nu$ are fixed to the values determined by the finite-size scaling analysis on the staggered magnetization $m_{\mathrm{s}}(u, L)$ (see Fig. 9). Thus, only $\eta_{\psi}$ is considered in the fitting procedure. The critical exponents and the system sizes $L$ used are indicated in the figures. The number in parentheses indicates the estimated error in the last digit.

in Eq. (10) by taking the simple power-law fit of $Z_{L}$ to extrapolate in the thermodynamic limit (see Figs. 4 and 6) [44]. We believe that the finite-size scaling analysis based on Eq. (12) results in a more accurate estimation of the exponent since all data, not only in the metallic region for $U<U_{\mathrm{c}}$ but also in the insulating region for $U>U_{\mathrm{c}}$, are used.

The critical exponents obtained are summarized in Table I for the honeycomb lattice model and the $\pi$-flux model. As clearly shown in Table I, these two different models lead to the same critical exponents for the spin and charge sectors with a considerable degree of accuracy. Therefore, our numerical results firmly verify the universal quantum criticality in the apparently different lattice models, which share only the massless Dirac energy dispersion in the noninteracting limit. To our knowledge, this represents one of the first unbiased studies on the critical properties of the metal-insulator transition in two spatial dimensions. The highly accurate estimation of the critical exponents, as well as the critical quantities, follows from the unprecedentedly large-scale simulations that we are now able to perform [14], combined with the careful and accurate finite-size scaling.

\section{Scaling functions}

In order to firmly establish the universal character of the metal-insulator transition, we follow Privman and Fisher's argument [62]. It states that if two different models belong to the same universality class, the scaling functions of a physical quantity for these two models are related by nonuniversal metric factors as

$$
f_{\alpha}(x)=c_{2} f_{\alpha}^{\prime}\left(c_{1} x\right),
$$

where $f_{\alpha}(x)$ and $f_{\alpha}^{\prime}(x)$ are the scaling functions for the two models. Figures 12(a) and 12(b) show the collapse fits
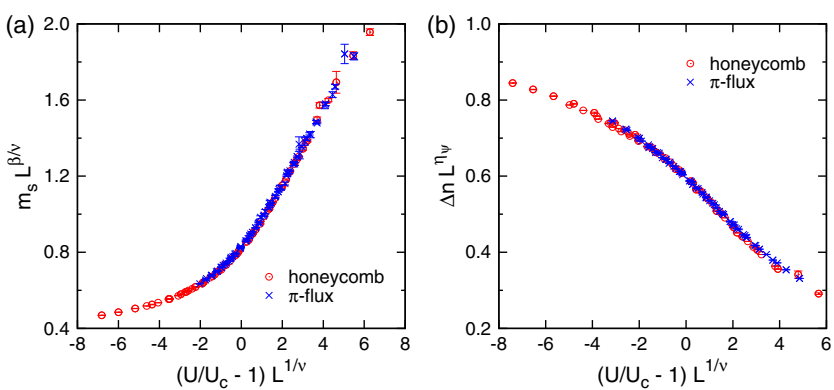

FIG. 12. Superposed scaling functions of the honeycomb lattice model (red circles) and the $\pi$-flux model (blue crosses) for (a) the staggered magnetization and (b) the jump in $n\left(\varepsilon_{k}\right)$. Data for the $\pi$-flux model are scaled with nonuniversal metric factors $c_{1}=0.768$ and $c_{2}=1.38$ for (a) and $c_{1}=0.851$ and $c_{2}=1.10$ for (b). The system sizes used are $L \geq 15$ for the honeycomb lattice model and $L \geq 20$ for the $\pi$-flux model.

without the correction term for the staggered magnetization and for the jump in $n\left(\varepsilon_{k}\right)$, respectively. We clearly find in Fig. 12 that the collapse fits for the honeycomb lattice model can superpose onto the appropriately scaled collapse fits for the $\pi$-flux model with the nonuniversal constants $c_{1}$ and $c_{2}$. This implies that the scaling functions of the two models are essentially equal and confirms, in a robust and unambiguous way, the existence of the universal critical behavior of the metal-insulator transition studied here.

\section{CHARGE STRUCTURE FACTOR}

Let us now investigate the long wavelength limit or, equivalently, the small $|\boldsymbol{q}|$ behavior of the static charge structure factor $N(\boldsymbol{q})$. Since the static structure factor $N(\boldsymbol{q})$ is given by the integral over all the frequencies of the dynamical structure factor $N(\boldsymbol{q}, \omega)$, i.e. $N(\boldsymbol{q})=\int d \omega N(\boldsymbol{q}, \omega)$, it depends, e.g., as in the spatial dimensionality $D>1$ within Fermi-liquid theory [63,64], on the charge and the Fermi velocities, as well as on other low-energy parameters such as the Landau parameter $F_{0}^{s}$ in the standard Fermi-liquid theory. Therefore, this is an interesting quantity, and it gives us information on how the dynamics evolves as we approach the metalinsulator transition point at $U_{\mathrm{c}}$ from the metallic side. The static charge structure factor $N(\boldsymbol{q})$ at momentum $\boldsymbol{q}$ is defined as

$$
N(\boldsymbol{q})=\frac{1}{N_{\mathrm{U}}} \sum_{j, k=1}^{N_{\mathrm{U}}} e^{i \boldsymbol{q} \cdot\left(\boldsymbol{r}_{j}-\boldsymbol{r}_{k}\right)}\left\langle n_{j} n_{k}\right\rangle,
$$

where $N_{\mathrm{U}}$ is the number of unit cells, $n_{j}=\sum_{\alpha} \sum_{s} c_{j \alpha s}^{\dagger} c_{j \alpha s}$, and $c_{j \alpha s}^{\dagger}$ is the creation operator of the electron at unit cell $\boldsymbol{r}_{j}$ and sublattice $\alpha(=A, B)$ with spin $s(=\uparrow, \downarrow)$. In the metallic region for $U<U_{\mathrm{c}}$, the charge structure factor should behave as the noninteracting one, i.e., 


$$
N(\boldsymbol{q}) \sim \alpha|\boldsymbol{q}|^{2} \ln |\boldsymbol{q}|
$$

for small $|\boldsymbol{q}|$, where $\alpha$ is a suitable constant that can be renormalized by the interaction, as in Fermi-liquid theory [63].

Figure 13 shows the ratio

$$
R=\frac{N\left(q^{*}\right)}{N_{U=0}\left(q^{*}\right)}
$$

of the charge structure factor for finite $U$ and the noninteracting limit, denoted as $N_{U=0}(\boldsymbol{q})$, at the smallest nonzero momentum $q^{*}$ available for a given system size. Since $N(\boldsymbol{q}) \simeq|\boldsymbol{q}|^{2}$ in the insulating phase [65-67], we expect that $R \sim 1 / \ln L$ in the insulating phase, whereas $R \sim$ const in the metallic phase. Therefore, there is a change of behavior in $R$ across the critical value of $U_{\mathrm{c}}$. Indeed, we find in Fig. 13 that $R$ decreases with increasing $L$ for $U$ larger than $U_{\mathrm{c}}$.

It should be emphasized that $R$ remains finite as we approach the transition point from the metallic side. As shown in Fig. 14, $R$ as a function of $U / t$ for different system sizes crosses around the critical point at a finite value of $R$, which indicates that the coefficient $\alpha$ in Eq. (15) is neither singular nor critical at the critical point for $U \rightarrow U_{\mathrm{c}}$, but approaches a finite constant in the thermodynamic limit. On the other hand, the coefficient $\alpha$ vanishes at the metal-insulator transition within the Gutwiller [7] or Brinkman-Rice [8] approximation on any lattice (including the honeycomb lattice) as a result of the vanishing of the double occupancy $\bar{d}=\left\langle n_{i \uparrow} n_{i \downarrow}\right\rangle$ and the sum rule valid at half-filling,

$$
\sum_{|\boldsymbol{q}| \neq 0} N(\boldsymbol{q})=N_{\mathrm{U}}\left(4 \bar{d}+2 g_{\mathrm{NN}}\right)
$$

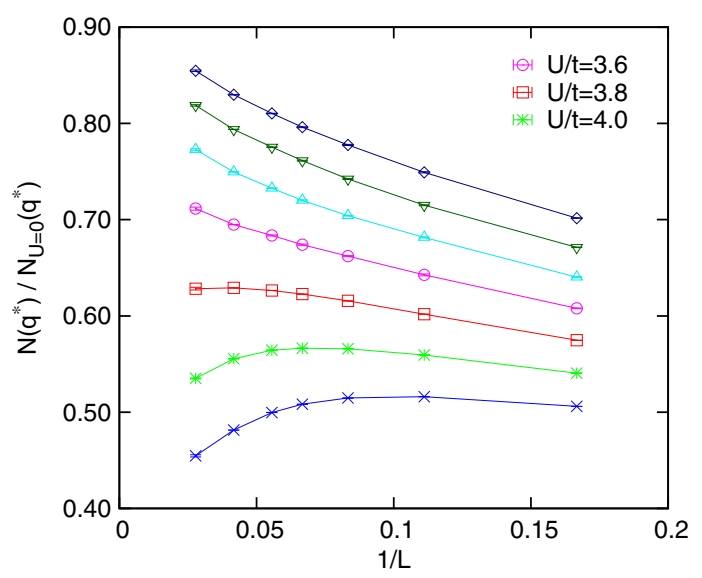

FIG. 13. Charge structure factors $N(\boldsymbol{q})$ at the smallest nonzero momentum $|\boldsymbol{q}|=q^{*}$ divided by the charge structure factor $N_{U=0}\left(q^{*}\right)$ in the noninteracting limit for the honeycomb lattice model. The interaction parameters are $U / t=3.0,3.2,3.4,3.6$, $3.8,4.0$, and 4.2 from top to bottom.

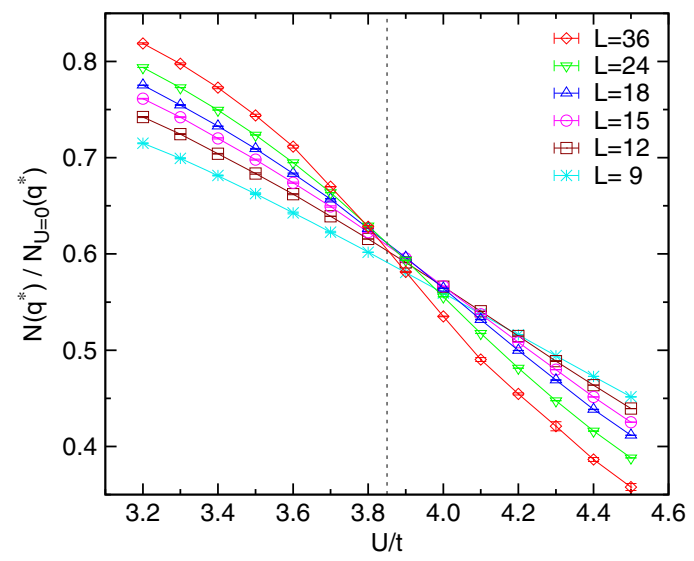

FIG. 14. Charge structure factors $N(\boldsymbol{q})$ at the smallest nonzero momentum $|\boldsymbol{q}|=q^{*}$ divided by the charge structure factor $N_{U=0}\left(q^{*}\right)$ in the noninteracting limit for the honeycomb lattice model with different system sizes indicated in the figure. The dashed line denotes the critical $U_{\mathrm{c}}$ determined by the datacollapse fit of the staggered magnetization in Fig. 9(a).

where $g_{\mathrm{NN}}$ is the nearest-neighbor density correlation, i.e., $g_{\mathrm{NN}}=\left\langle n_{j A} n_{j B}\right\rangle-\left\langle n_{j A}\right\rangle\left\langle n_{j B}\right\rangle$, and $n_{j \alpha}=\sum_{s} c_{j \alpha s}^{\dagger} c_{j \alpha s}$. This is simply because $g_{\mathrm{NN}} \rightarrow 0$ when $\bar{d} \rightarrow 0$ at the metalinsulator transition described by the Gutzwiller approximation [68], and is therefore in sharp contrast with our results.

Although our results do not represent direct evidence for the nonsingular behavior of $v_{\mathrm{F}}$ close to the metal-insulator transition, $v_{\mathrm{F}}$ is certainly related to the charge velocity, as in Fermi-liquid theory, which in turn affects the value of $\alpha$. Therefore, our results imply that $v_{\mathrm{F}}$ remains finite at the metal-insulator transition. In this respect, it should be noted that the evolution of $\alpha$ as a function of $U$ found in Fig. 14 is compatible to the expected behavior of the Dirac Fermi velocity $v_{\mathrm{F}}$ for the GN universality class of the metalinsulator transition [34]. As discussed previously [36], the vanishing of the quasiparticle weight $Z$ without a renormalization of $v_{\mathrm{F}}$ is understood as a consequence of an equal divergence in the momentum $\boldsymbol{k}$ and frequency $\omega$ derivatives of the electron self-energy $\Sigma(\boldsymbol{k}, \omega)$ at the Dirac point. The quasiparticle weight $Z$ at the Dirac point with momentum $\boldsymbol{k}_{\mathrm{F}}$ is given as

$$
Z=\left[1-\left.\frac{\partial}{\partial \omega} \operatorname{Re} \Sigma\left(\boldsymbol{k}_{\mathrm{F}}, \omega\right)\right|_{\omega=0}\right]^{-1},
$$

while the Dirac Fermi velocity $v_{\mathrm{F}}$ is related to $Z$ as

$$
\frac{v_{\mathrm{F}}}{v_{\mathrm{F}}^{0}}=Z\left[1+\left.\frac{1}{v_{\mathrm{F}}^{0}} \frac{\partial}{\partial k} \operatorname{Re} \Sigma(\boldsymbol{k}, 0)\right|_{\boldsymbol{k}=\boldsymbol{k}_{\mathrm{F}}}\right] .
$$

Therefore, in order to compensate for the divergence of $\left.(\partial / \partial \omega) \operatorname{Re} \Sigma\left(\boldsymbol{k}_{\mathrm{F}}, \omega\right)\right|_{\omega=0}$, i.e., $Z \rightarrow 0$, at the metal-insulator transition point, $\left.(\partial / \partial k) \operatorname{Re} \Sigma(\boldsymbol{k}, 0)\right|_{k=k_{\mathrm{F}}}$ must diverge at the 
same time. This implies that the strong momentum dependence of $\Sigma(\boldsymbol{k}, \omega)$ around the Dirac point, not included in the simplest DMFT approach, is an essential ingredient to describe the metal-insulator transition. Similar arguments are also found in earlier studies of the $t-J$ model with the large- $N$ expansion [70]. It is also interesting to note that the nodal Fermi velocity remains finite in the carriernumber-controlled Mott metal-insulator transition described by a Gutzwiller projected $d$-wave BCS state, which exhibits the massless Dirac dispersion at the nodal point [71].

In any event, our results are certainly useful to characterize the metal-insulator transition in the charge sector. Indeed, the critical value $U_{\mathrm{c}}$ in the charge sector can be estimated directly by this simple analysis shown in Fig. 14 without performing rather elaborated finite-size scaling of quantities such as the charge gap [12,19], which is usually very time consuming and difficult to compute with high accuracy.

\section{DISCUSSION AND CONCLUSIONS}

The GN models have been extensively studied in quantum field theory [23]. In the standard field theoretical treatment of the transition, the critical behavior is studied in space-time dimension $d=D+1$, where $D$ is the spatial dimensionality. The critical exponents of the GN models have been evaluated by several standard techniques, such as the large- $N$ expansion $[51,54]$ and the $\epsilon$ expansion around the lower $d=2+\epsilon$ [53] or upper $d=4-\epsilon$ [31] critical dimensions, and these are summarized in Table I. It is clearly noticed in Table I that there exist sizable discrepancies among the critical exponents calculated by these different analytical techniques. Therefore, an unbiased numerical study is highly desired to clarify the critical behavior of the GN models.

In this paper, we have established, based on robust and reliable numerical simulations on fairly large clusters, the universal properties of the metal-insulator transition for the two different lattice models of interacting Dirac electrons in two spatial dimensions. We have determined the critical exponents that characterize the universal quantum critical behavior in both metallic and insulating phases at the vicinity of the transition for these models. Since it is expected that the effective low-energy theory of these lattice models is described in the continuous limit by the chiral Heisenberg universality class of the GN model with $N=8$, our study also currently represents the most accurate and reliable results for this fundamental GN model to reveal the universal critical behavior. Indeed, our results resolve some of the inconsistency among different approximate approaches for the GN models shown in Table I, especially evident for $N=8$.

We have also clarified how the quasiparticle in the SM phase collapses as the AF insulating phase is approached with increasing $U$ for these two models. We have shown that the quasiparticle weight $Z$ diminishes and becomes zero at the metal-insulator transition, and we found that the exponent $\eta_{Z}=\nu \eta_{\psi}$, characterizing the renormalization of the quasiparticle weight $Z$ [see Eq. (10)], is much smaller for both models $\left(\eta_{Z} \simeq 0.2\right)$ than the one predicted by simple mean-field and dynamical mean-field correlated theories of the metal-insulator transition, for which $\eta_{Z}=1[8,10]$. More interestingly, we have also found that the small $q$ limit of the static charge structure factor is not singular at the metal-insulator transition, suggesting that the Dirac Fermi velocity $v_{\mathrm{F}}$ is not critical at the transition. These critical behaviors, small $\eta_{Z}$ and finite $v_{\mathrm{F}}$, are qualitatively the same as the ones expected for the GN universality class of the metal-insulator transition [34]. Therefore, our results provide clear numerical evidence that the critical behavior of the GN model also applies to lattice models and correctly describes the metal-insulator transition in interacting Dirac electrons. It should be noted here that the noncriticality of $v_{\mathrm{F}}$ for the honeycomb lattice model is also found by quantum cluster methods [35,36], although it was first overlooked within the single-site DMFT [33].

Strictly speaking, the metal-insulator transition studied here does not describe a genuine Mott transition because the insulating phase breaks $S U(2)$ symmetry for $U>U_{\mathrm{c}}$. Indeed, as discussed below, each type of possible symmetry breaking in interacting Dirac fermions determines a different universality class of the transition characterized with different critical exponents. Nevertheless, the metalinsulator transition studied here does not originate from a Slater-type nesting instability at weak coupling since the density of states is zero at the Fermi level. The transition instead occurs at an intermediate- or strong-coupling region as evidenced by the fact that $U_{\mathrm{c}} \simeq 5.55 t$ for the $\pi$-flux model is almost the same as the noninteracting bandwidth $4 \sqrt{2} t$.

Very recently, using the continuous-time QMC, Wang et al. [58] studied similar models of spinless fermions with the nearest-neighbor repulsion $V$, where, by increasing $V$, a transition from the SM to a staggered charge-density-wave (CDW) state occurs. With a finite-size scaling analysis based on the results for clusters up to 450 sites, they have estimated the critical exponents, $\nu$ and $\beta$, for the CDW order parameter [58]. These critical exponents have recently been revisited by the Majorana QMC method on larger clusters up to 1152 sites [59] and also by finite-temperature simulations $[60,72]$. As seen in Table I, these exponents are clearly different from those for the spinful models that we have studied here. This is simply understood because these spinless and spinful models belong to different universality classes. Indeed, it is known that the spinless lattice models with the nearest-neighbor interaction at halffilling are described in the continuous limit by the GN model with $N=4$ and the chiral Ising universality class. It is also interesting to notice in Table I that the critical exponents for the spinless lattice models estimated numerically are rather different from the analytical results. This also demonstrates that numerically exact studies are highly valuable to accurately determine the quantum criticality 
and to remove the ambiguities that might arise from inadequate approximations.

It should be emphasized that the various universality classes depend on the physics, namely, the symmetry that is broken in the ordered phase and the total number $N$ of Dirac fermion components that describe the corresponding critical theory [52]. The well-explored universality classes for the GN models in the continuous limit include the following three classes:

(1) Chiral Ising universality class. $Z_{2}$ symmetry, i.e., a discrete order parameter, is broken, for instance, when a commensurate CDW order settles.

(2) Chiral $X Y$ universality class. $U(1)$ symmetry is broken and the order parameter is characterized by an angle, as in a superconducting state.

(3) Chiral Heisenberg universality class. $S U(2)$ symmetry is broken. This should occur in the transition studied here, as the order parameter - the staggered magnetization - is characterized by a vector with three components [SU(2) is equivalent to $S O(3)]$.

Among these, the two classes have been studied so far based on the lattice models with unbiased numerical techniques: the chiral Ising universality class by Wang et al. [58] and Li et al. [59], and the chiral Heisenberg universality class studied here and in Ref. [18]. We expect that the quantum criticality belonging to the chiral $X Y$ universality class emerges in a negative $U$ Hubbard model with the Dirac points of the noninteracting energy dispersion at the Fermi level, provided that the $S U(2)$ symmetry that relates the CDW order to the superconducting one is not satisfied (otherwise, the chiral Heisenberg universality class applies again). For example, by adding the next-nearest-neighbor hopping $t^{\prime}$ in the same lattice models studied here but with a negative $U$ (no sign problem with $t^{\prime}$ in the negative $U$ Hubbard model), the chiral $X Y$ universality class with different critical exponents can be investigated in the same unbiased numerical approach.

In conclusion, we have investigated the critical behaviors of the metal-insulator transition in the interacting Dirac electrons in two spatial dimensions, described by the two different lattice models, the Hubbard model on the honeycomb lattice and the $\pi$-flux Hubbard model on the square lattice, at half-filling. We have performed the unprecedentedly large-scale quantum Monte Carlo simulations to systematically calculate the staggered magnetization and the momentum distribution function. The calculation of the momentum distribution function is particularly important because it allows us to examine the quasiparticle weight and thus also explore the critical behavior in the metallic side, which has never been successful previously in the unbiased numerically exact calculations. The ground-state phase diagrams determined by extrapolating the staggered magnetization and the quasiparticle weight to the thermodynamic limit have revealed the continuous nature of the transition between the SM and the AF insulator with no intermediate phase. Therefore, our results firmly rule out the possibility of a spin-liquid phase for these two models proposed in the earlier studies. We have obtained the critical exponents by careful and accurate finite-size scaling analysis and found that the two lattice models belong to the same universality class. Since the low-energy effective model in the continuous limit for these two models is described by the GN model with $N=8$ and the chiral Heisenberg universality class, our results currently represent the most accurate determination of the quantum criticality of this universality class. Finally, we have shown that the quasiparticle weight monotonically decreases with increasing $U$ and becomes zero at the metal-insulator transition, while the Fermi velocity seems noncritical. This qualitatively important feature is indeed in good agreement with the one expected for the GN universality class of the metal-insulator transition and cannot be captured by the simple mean-field or Gutzwiller-type approximate argument.

\section{ACKNOWLEDGMENTS}

We acknowledge B. Rosenstein for useful discussions and for clarifying the notations in Ref. [31]. We are also grateful to I. F. Herbut, A. Gambassi, M. Capone, K. Harada, F. Parisen Toldin, T. Sato, and Y. Hatsugai for valuable comments. This work has been supported in part by the Grant-in-Aid for Scientific Research from MEXT Japan (under Grants No. 24300020 and No. 26400413), RIKEN iTHES Project and Molecular Systems, MIUR-PRIN-2010, and MEXT HPCI Strategic Programs for Innovative Research (SPIRE) (Grant No. hp140168). The numerical simulations have been performed on K computer at RIKEN Advanced Institute for Computational Science (AICS), RIKEN Cluster of Clusters (RICC), RIKEN supercomputer system (HOKUSAI GreatWave), and NEC SX-ACE at Cybermedia Center, Osaka University.

\section{APPENDIX A: ENERGY-RESOLVED MOMENTUM DISTRIBUTION FUNCTION}

The quasiparticle weight can be, in general, calculated from the jump in the momentum distribution function at the Fermi level. For the honeycomb lattice model and the $\pi$-flux model studied here, the momentum distribution function $n_{\alpha \beta, s}(\boldsymbol{k})$ at momentum $\boldsymbol{k}$ is defined as

$$
n_{\alpha \beta, s}(\boldsymbol{k})=\left\langle c_{\boldsymbol{k} \alpha s}^{\dagger} c_{\boldsymbol{k} \beta s}\right\rangle,
$$

where

$$
c_{\boldsymbol{k} \alpha s}^{\dagger}=\frac{1}{\sqrt{N_{\mathrm{U}}}} \sum_{r} e^{-i \boldsymbol{k} \cdot \boldsymbol{r}} c_{r \alpha s}^{\dagger},
$$

with $c_{r \alpha s}^{\dagger}$ the creation operator of the electron at unit cell $\boldsymbol{r}$ and sublattice $\alpha(=A, B)$ with spin $s=(\uparrow, \downarrow)$, and $N_{\mathrm{U}}$ the number of unit cells. Notice that, because of the particlehole symmetry at half-filling, $n_{A A, s}(\boldsymbol{k})=n_{B B, s}(\boldsymbol{k})=1 / 2$ for all momenta, and therefore only $n_{A B, s}(\boldsymbol{k})$ and $n_{B A, S}(\boldsymbol{k})$ are nontrivial with $\left[n_{A B, S}(\boldsymbol{k})\right]^{*}=n_{B A, S}(\boldsymbol{k})$. 
The energy-resolved momentum distribution function $n\left(\varepsilon_{k}\right)$ is defined as

$$
n\left(\varepsilon_{\boldsymbol{k}}\right)=\left\langle\psi_{\boldsymbol{k}, \pm, s}^{\dagger} \psi_{\boldsymbol{k}, \pm, s}\right\rangle,
$$

where the annihilation operators $\psi_{k,-, s}$ and $\psi_{k,+, s}$ of the bonding and antibonding states, respectively, are given as

$$
\psi_{k, \pm, s}=\frac{1}{\sqrt{2}}\left(c_{k A s} \pm \frac{h_{k}}{\left|h_{k}\right|} c_{k B s}\right) .
$$

The states $\psi_{k, \pm, s}$ are the eigenstates of the noninteracting Hamiltonian

$$
H_{0}=\sum_{k, s}\left(\begin{array}{ll}
c_{k A s}^{\dagger} & c_{k B S}^{\dagger}
\end{array}\right)\left(\begin{array}{cc}
0 & h_{k} \\
h_{\boldsymbol{k}}^{*} & 0
\end{array}\right)\left(\begin{array}{c}
c_{k A s} \\
c_{\boldsymbol{k} B S}
\end{array}\right)
$$

at momentum $\boldsymbol{k}$ with the energy $\varepsilon_{\boldsymbol{k}}= \pm\left|h_{\boldsymbol{k}}\right|$. Here,

$$
h_{k}=-t\left(1+e^{-i \boldsymbol{k} \cdot \vec{\tau}_{1}}+e^{-i \boldsymbol{k} \cdot \vec{\tau}_{2}}\right)
$$

for the honeycomb lattice model and

$$
h_{\boldsymbol{k}}=-t\left(1+e^{-i \boldsymbol{k} \cdot \vec{\tau}_{1}}+e^{-i \boldsymbol{k} \cdot \vec{\tau}_{2}}-e^{-i \boldsymbol{k} \cdot\left(\vec{\tau}_{1}+\vec{\tau}_{2}\right)}\right)
$$

for the $\pi$-flux model, where $\vec{\tau}_{1}$ and $\vec{\tau}_{2}$ are the primitive translational vectors defined in Figs. 1(a) and 1(b).

In principle, in order to determine the quasiparticle weight $Z$, the occupation number should be calculated in terms of the natural orbitals, i.e., the eigenvectors of the density matrix. To this end, we note that the density matrix at momentum $\boldsymbol{k}$ is a $2 \times 2$ matrix and is represented as

$$
\left(\begin{array}{ll}
n_{A A, S}(\boldsymbol{k}) & n_{A B, S}(\boldsymbol{k}) \\
n_{B A, S}(\boldsymbol{k}) & n_{B B, S}(\boldsymbol{k})
\end{array}\right)=\left(\begin{array}{cc}
1 / 2 & f_{\boldsymbol{k}} \\
f_{\boldsymbol{k}}^{*} & 1 / 2
\end{array}\right) .
$$

Therefore, the "dressed" quasiparticle operators simply read

$$
\bar{\psi}_{\boldsymbol{k}, \pm, s}=\frac{1}{\sqrt{2}}\left(c_{k A s} \pm \frac{f_{\boldsymbol{k}}^{*}}{\left|f_{\boldsymbol{k}}\right|} c_{\boldsymbol{k} B s}\right)
$$

with the occupation

$$
\left\langle\bar{\psi}_{\boldsymbol{k}, \pm, s}^{\dagger} \bar{\psi}_{\boldsymbol{k}, \pm, s}\right\rangle=\frac{1}{2} \pm\left|f_{\boldsymbol{k}}\right| .
$$

Notice that $f_{k}=-\frac{1}{2}\left(h_{k}^{*} /\left|h_{k}\right|\right)$ in the noninteracting limit where the bonding (antibonding) states are all occupied (empty). We have verified that even in the interacting case studied here, the natural orbitals $\bar{\psi}_{\boldsymbol{k}, \pm, s}$ are almost indistinguishable from the noninteracting bonding and antibonding states $\psi_{\boldsymbol{k}, \pm, s}$. Indeed, we find in Fig. 15 that the difference between the quasiparticle weight $Z$ calculated with the natural orbitals and the one with the noninteracting bonding and antibonding states is negligible for the models studied here, both in the metallic and in the insulating
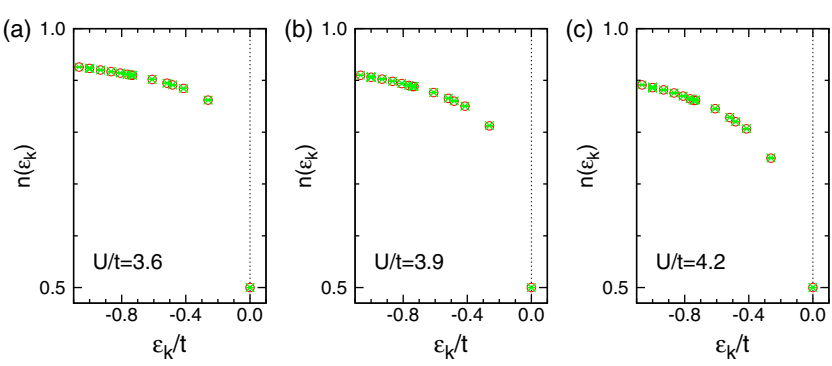

FIG. 15. Energy-resolved momentum distribution function $n\left(\varepsilon_{\boldsymbol{k}}\right)$ below the Fermi level $\left(\varepsilon_{\boldsymbol{k}}=0\right)$ for the honeycomb lattice model with $L=24$ and different values of $U / t$ indicated in the figures. Red circles and green crosses represent the results calculated using the noninteracting bonding states [Eq. (A4)] and the natural orbitals [Eq. (A9)], respectively.

phases. Therefore, the treatment for computing the quasiparticle weight $Z$ in Secs. III B and IV B, i.e., the jump of the energy-resolved momentum distribution function $n\left(\varepsilon_{k}\right)$ at the Fermi level, is not only asymptotically valid to determine the corresponding critical exponent but also represents a good quantitative estimate of $Z$.

\section{APPENDIX B: EFFECTS OF THE LEADING CORRECTION TERM IN THE FINITE-SIZE SCALING ANALYSES}

Here, we examine the robustness of the fitting parameters in Eqs. (11) and (12) with and without the leading correction term in the finite-size scaling analyses. Since the leading correction term is expected to be less important for sufficiently large clusters, we examine the system-size dependence of the fitting parameters.

Tables II and III summarize the fitting parameters in the staggered magnetization, which are obtained for clusters including the smallest lattice size $L_{\min }$ in the data collapse

TABLE II. Results of the critical points $U_{\mathrm{c}} / t$ and the critical exponents $\nu$ and $\beta$ obtained from collapsing data of the staggered magnetization, $m_{\mathrm{s}}(u, L)$, without the leading correction term, i.e., $c=0$ in Eq. (11), for the honeycomb lattice model (upper rows) and the $\pi$-flux model (lower rows). $L_{\min }$ refers to the smallest $L$ used in the data collapse. The maximum $L$ is 36 for the honeycomb lattice model and 40 for the $\pi$-flux model.

\begin{tabular}{llll}
\hline \hline$L_{\min }$ & $U_{\mathrm{c}} / t$ & \multicolumn{1}{c}{$\nu$} & \multicolumn{1}{c}{$\beta$} \\
\hline 6 & $3.782(3)$ & $1.025(4)$ & $0.881(4)$ \\
9 & $3.785(5)$ & $1.037(5)$ & $0.886(6)$ \\
12 & $3.800(6)$ & $1.041(5)$ & $0.876(7)$ \\
15 & $3.820(7)$ & $1.038(7)$ & $0.856(10)$ \\
18 & $3.833(9)$ & $1.040(11)$ & $0.841(14)$ \\
8 & $5.415(10)$ & $0.999(9)$ & $0.873(9)$ \\
12 & $5.418(14)$ & $1.018(10)$ & $0.886(12)$ \\
16 & $5.455(21)$ & $1.011(11)$ & $0.861(17)$ \\
20 & $5.509(24)$ & $1.025(12)$ & $0.838(20)$ \\
24 & $5.511(37)$ & $1.053(22)$ & $0.855(36)$ \\
\hline \hline
\end{tabular}


TABLE III. Same as Table II, but with the leading correction term $c L^{-\omega}(c \neq 0)$ in Eq. (11).

\begin{tabular}{lcccc}
\hline \hline$L_{\min }$ & $U_{\mathrm{c}} / t$ & $\nu$ & $\beta$ & $\omega$ \\
\hline 6 & $3.843(8)$ & $1.005(5)$ & $0.74(2)$ & $0.55(4)$ \\
9 & $3.858(9)$ & $1.012(5)$ & $0.74(2)$ & $0.78(5)$ \\
12 & $3.856(10)$ & $1.020(7)$ & $0.75(2)$ & $0.91(5)$ \\
15 & $3.853(10)$ & $1.021(8)$ & $0.75(2)$ & $0.89(6)$ \\
18 & $3.849(10)$ & $1.028(10)$ & $0.76(2)$ & $0.82(12)$ \\
8 & $5.423(38)$ & $0.998(10)$ & $0.86(5)$ & $0.17(35)$ \\
12 & $5.534(41)$ & $1.007(10)$ & $0.76(5)$ & $0.94(25)$ \\
16 & $5.557(31)$ & $1.008(11)$ & $0.74(3)$ & $1.02(13)$ \\
20 & $5.546(27)$ & $1.021(11)$ & $0.76(3)$ & $0.85(24)$ \\
24 & $5.537(35)$ & $1.050(19)$ & $0.78(4)$ & $0.83(17)$ \\
\hline \hline
\end{tabular}

without and with the leading correction term in the finitesize scaling ansatz of Eq. (11), respectively. These results are also compared in Fig. 16 as a function of $L_{\min }^{-1}$. We notice that $U_{\mathrm{c}} / t$ systematically increases as the data sets with smaller $L$ are removed when we use the finite-size scaling ansatz without the leading correction term, i.e., $c=0$ in Eq. (11). Accordingly, the critical exponent $\beta$ tends to decrease, although the critical exponent $\nu$ is less affected by including or not including the leading correction term in the finite-size scaling ansatz, at least, within the statistical errors. These systematic differences with varying
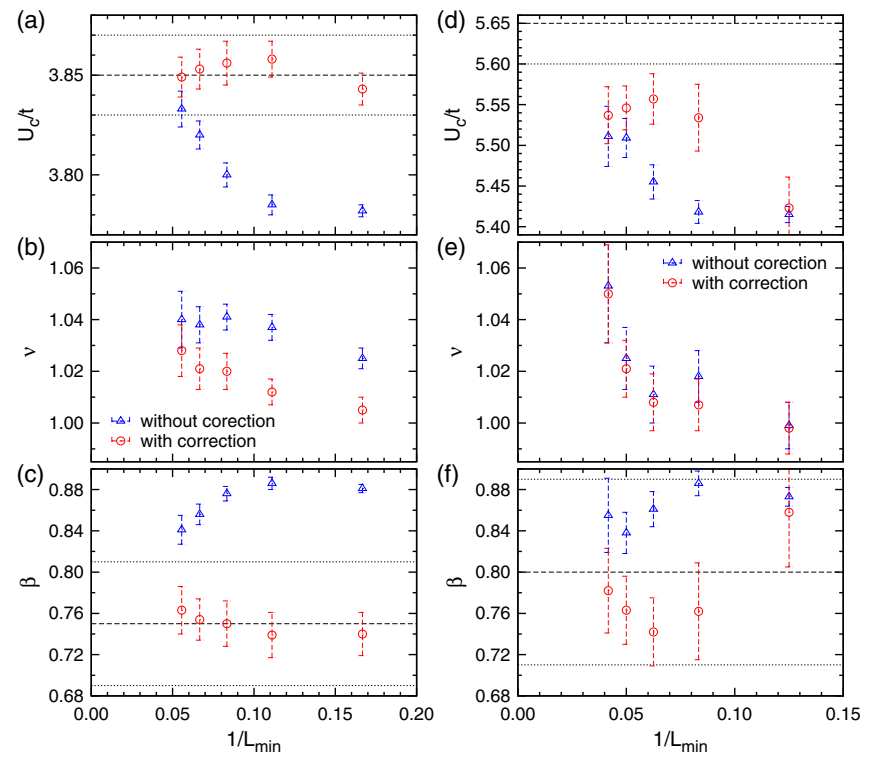

FIG. 16. Same data as in Tables II and III plotted as a function of $1 / L_{\min }$ for the honeycomb lattice model (left panels) and the $\pi$-flux model (right panels). Red circles (blue triangles) represent the results with (without) the leading correction term in Eq. (11). For comparison, $U_{\mathrm{c}} / t$ and $\beta$ estimated directly from the AF order parameter $m_{\mathrm{s}}$ in Fig. 4 are indicated by dashed lines (with the statistical errors denoted by dotted lines) in (a), (c), (d), and (f). Notice that these two different estimations of $U_{\mathrm{c}} / t$ and $\beta$ provide statistically consistent results.
TABLE IV. Results of the critical exponent $\eta_{\psi}$ obtained from collapsing data of the jump in the energy-resolved momentum distribution function $\Delta n(u, L)$ without the leading correction term, i.e., $d=0$ in Eq. (B1), for the honeycomb lattice model (left rows) and the $\pi$-flux model (right rows). $L_{\min }$ refers to the smallest $L$ used in the data collapse. The maximum $L$ is 36 for the honeycomb lattice model and 40 for the $\pi$-flux model.

\begin{tabular}{lcccc}
\hline \hline \multirow{2}{*}{ Honeycomb lattice } & & \multicolumn{2}{c}{$\pi$-flux model } \\
\cline { 5 - 5 } \cline { 5 - 5 }$L_{\text {min }}$ & $\eta_{\psi}$ & & $L_{\min }$ & $\eta_{\psi}$ \\
\hline 6 & $0.17(2)$ & & 8 & $0.19(2)$ \\
9 & $0.18(2)$ & & 12 & $0.21(2)$ \\
12 & $0.19(2)$ & & 16 & $0.22(2)$ \\
15 & $0.20(2)$ & & 20 & $0.23(2)$ \\
18 & $0.20(2)$ & & 24 & $0.24(2)$ \\
\hline \hline
\end{tabular}

$L_{\min }$ imply that the leading correction term in the finite-size scaling ansatz is not negligible.

On the other hand, when the leading correction term is included, the results are robust against the choice of $L_{\min }$, as shown in Table III and Fig. 16. In addition, $U_{\mathrm{c}}$ and $\beta$ evaluated in the data-collapse analysis are statistically consistent with those obtained by the direct fit of thermodynamically extrapolated staggered magnetization, shown in Fig. 4. In Sec. IVA, we report the results of the data collapses with the leading correction term because they are clearly more accurate and stable. The quality of our data and extrapolations is further supported by the fact that both results obtained with and without the leading correction term tend to be identical when the system sizes are large enough, as shown in Tables II and III, and in Fig. 16.

The finite-size scaling ansatz for the jump of $n\left(\varepsilon_{k}\right)$, including the leading correction term, is given as

$$
\Delta n(u, L)=L^{-\eta_{\psi}}\left(1+d L^{-\omega^{\prime}}\right) f_{n}\left(u L^{1 / \nu}\right)
$$

where $d$ and $\omega^{\prime}$ are additional fitting parameters. The obtained critical exponents $\eta_{\psi}$ for various $L_{\min }$ are summarized in Tables IV and V, and also in Fig. 17. We find that the stable solutions with $\omega^{\prime}>0$ cannot be obtained for small $L_{\min }$. Moreover, the estimated $\omega^{\prime}$ tends to increase for larger $L_{\text {min }}$ (see Table V), and it is not possible to reach a converged value of $\omega^{\prime}$ within the given statistical accuracy and the system sizes available.

TABLE V. Same as Table IV, but with the leading correction term $d L^{-\omega^{\prime}}(d \neq 0)$ in Eq. (B1).

\begin{tabular}{lcclllll}
\hline \hline \multicolumn{3}{c}{ Honeycomb lattice } & & \multicolumn{3}{c}{$\pi$-flux model } \\
\cline { 1 - 2 } \cline { 5 - 6 }$L_{\text {min }}$ & $\eta_{\psi}$ & $\omega^{\prime}$ & & $L_{\text {min }}$ & $\eta_{\psi}$ & $\omega^{\prime}$ \\
\hline 12 & $0.25(5)$ & $1.3(6)$ & & 16 & $0.41(10)$ & $0.5(6)$ \\
15 & $0.23(3)$ & $1.6(3)$ & & 20 & $0.33(8)$ & $1.0(5)$ \\
18 & $0.23(3)$ & $1.7(3)$ & & 24 & $0.31(4)$ & $1.2(3)$ \\
\hline \hline
\end{tabular}



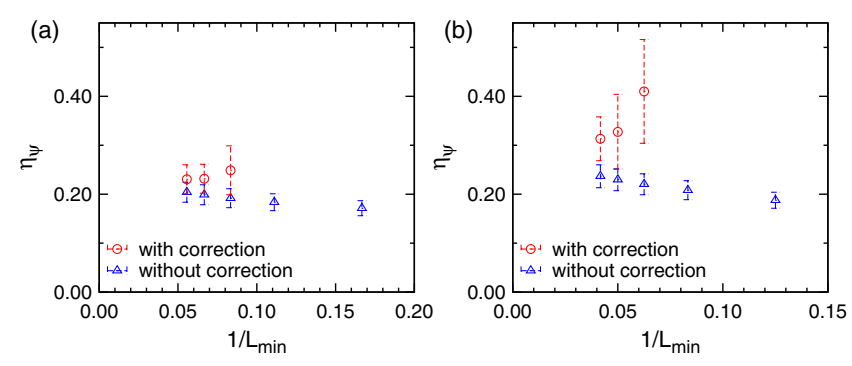

FIG. 17. Same data as in Tables IV and V plotted as a function of $1 / L_{\min }$ for (a) the honeycomb lattice model and (b) the $\pi$-flux model. Red circles (blue triangles) represent the results with (without) the leading correction term in Eq. (B1).

Nevertheless, we confirm that the estimated values of $\eta_{\psi}$ with the correction term are instead converged and fully consistent with those obtained without the correction term for large enough $L_{\min }$, as shown in Fig. 17. Therefore, in Sec. IV B, we only show the results of the data-collapse analysis without the leading correction term.

[1] L. Hoddeson, G. Baym, and M. Eckert, The Development of the Quantum-Mechanical Electron Theory of Metals: 1928-1933, Rev. Mod. Phys. 59, 287 (1987).

[2] M. Imada, A. Fujimori, and Y. Tokura, Metal-Insulator Transitions, Rev. Mod. Phys. 70, 1039 (1998).

[3] C. Kittel, Introduction to Solid State Physics (Wiley, New York, 1967).

[4] N. F. Mott, The Basis of the Electron Theory of Metals, with Special Reference to the Transition Metals, Proc. Phys. Soc. London Sect. A 62, 416 (1949).

[5] T. Giamarchi, Quantum Physics in One Dimension (Oxford University Press, Oxford, 2004).

[6] W. Metzner and D. Vollhardt, Correlated Lattice Fermions in $d=\infty$ Dimensions, Phys. Rev. Lett. 62, 324 (1989).

[7] M. Gutzwiller, Correlation of Electrons in a Narrow $s$ Band, Phys. Rev. 137, A1726 (1965).

[8] W. F. Brinkman and T. M. Rice, Application of Gutzwiller's Variational Method to the Metal-Insulator Transition, Phys. Rev. B 2, 4302 (1970).

[9] A. Georges, W. Krauth, and M. J. Rozenberg, Dynamical Mean-Field Theory of Strongly Correlated Fermion Systems and the Limit of Infinite Dimensions, Rev. Mod. Phys. 68, 13 (1996).

[10] G. Moeller, Q. Si, G. Kotliar, M. Rozenberg, and D. S. Fisher, Critical Behavior of the Mott Transition in the Hubbard Model, Phys. Rev. Lett. 74, 2082 (1995).

[11] For instance, in the square lattice with the nearest-neighbor hopping, the perfectly nested Fermi surface induces an insulating phase once nonzero $U$ is introduced.

[12] Z. Y. Meng, T. C. Lang, S. Wessel, F. F. Assaad, and A. Muramatsu, Quantum Spin Liquid Emerging in Two-Dimensional Correlated Dirac Fermions, Nature (London) 464, 847 (2010).

[13] Q. Chen, G. H. Booth, S. Sharma, G. Knizia, and G. K.-L. Chan, Intermediate and Spin-Liquid Phase of the
Half-Filled Honeycomb Hubbard Model, Phys. Rev. B 89, 165134 (2014).

[14] S. Sorella, Y. Otsuka, and S. Yunoki, Absence of a Spin Liquid Phase in the Hubbard Model on the Honeycomb Lattice, Sci. Rep. 2, 992 (2012).

[15] F. F. Assaad and I. F. Herbut, Pinning the Order: The Nature of Quantum Criticality in the Hubbard Model on Honeycomb Lattice, Phys. Rev. X 3, 031010 (2013).

[16] S. R. Hassan and D. Sénéchal, Absence of Spin Liquid in Nonfrustrated Correlated Systems, Phys. Rev. Lett. 110, 096402 (2013).

[17] D. Ixert, F. F. Assaad, and K. P. Schmidt, Mott Physics in the Half-Filled Hubbard Model on a Family of Vortex-Full Square Lattices, Phys. Rev. B 90, 195133 (2014).

[18] F. Parisen Toldin, M. Hohenadler, F. F. Assaad, and I. F. Herbut, Fermionic Quantum Criticality in Honeycomb and $\pi$-flux Hubbard Models: Finite-Size Scaling of Renormalization-Group-Invariant Observables from Quantum Monte Carlo, Phys. Rev. B 91, 165108 (2015).

[19] C.-C. Chang and R. T. Scalettar, Quantum Disordered Phase near the Mott Transition in the Staggered-Flux Hubbard Model on a Square Lattice, Phys. Rev. Lett. 109, 026404 (2012).

[20] I. Affleck and J.B. Marston, Large-n Limit of the Heisenberg-Hubbard Model: Implications for High- $T_{\mathrm{c}}$ Superconductors, Phys. Rev. B 37, 3774 (1988).

[21] I. Affleck, Z. Zou, T. Hsu, and P. W. Anderson, $S U(2)$ Gauge Symmetry of the Large-U Limit of the Hubbard Model, Phys. Rev. B 38, 745 (1988).

[22] Y. Otsuka, S. Yunoki, and S. Sorella, Mott Transition in the 2D Hubbard Model with $\pi$-flux, JPS Conf. Proc. 3, 013021 (2014).

[23] D. Gross and A. Neveu, Dynamical Symmetry Breaking in Asymptotically Free Field Theories, Phys. Rev. D 10, 3235 (1974).

[24] I. F. Herbut, Interactions and Phase Transitions on Graphene's Honeycomb Lattice, Phys. Rev. Lett. 97, 146401 (2006).

[25] I. F. Herbut, V. Juričić, and O. Vafek, Relativistic Mott Criticality in Graphene, Phys. Rev. B 80, 075432 (2009).

[26] R. Blankenbecler, D. J. Scalapino, and R. L. Sugar, Monte Carlo Calculations of Coupled Boson-Fermion Systems. I, Phys. Rev. D 24, 2278 (1981).

[27] J. E. Hirsch, Two-Dimensional Hubbard Model: Numerical Simulation Study, Phys. Rev. B 31, 4403 (1985).

[28] S. R. White, D. J. Scalapino, R. L. Sugar, E. Y. Loh, J. E. Gubernatis, and R. T. Scalettar, Numerical Study of the Two-Dimensional Hubbard Model, Phys. Rev. B 40, 506 (1989).

[29] S. Sorella and E. Tosatti, Semi-Metal-Insulator Transition of the Hubbard Model in the Honeycomb Lattice, Europhys. Lett. 19, 699 (1992).

[30] Y. Otsuka and Y. Hatsugai, Mott Transition in the TwoDimensional Flux Phase, Phys. Rev. B 65, 073101 (2002).

[31] B. Rosenstein and A. Kovner, Critical Exponents of New Universality Classes, Phys. Lett. B 314, 381 (1993).

[32] L. Janssen and I. F. Herbut, Antiferromagnetic Critical Point on Graphene's Honeycomb Lattice: A Functional Renormalization Group Approach, Phys. Rev. B 89, 205403 (2014). 
[33] M. T. Tran and K. Kuroki, Finite-Temperature SemimetalInsulator Transition on the Honeycomb Lattice, Phys. Rev. B 79, 125125 (2009).

[34] I. F. Herbut, V. Juričić, and B. Roy, Theory of Interacting Electrons on the Honeycomb Lattice, Phys. Rev. B 79, 085116 (2009).

[35] W. Wu, Y.-H. Chen, H.-S. Tao, N.-H. Tong, and W.-M. Liu, Interacting Dirac Fermions on Honeycomb Lattice, Phys. Rev. B 82, 245102 (2010).

[36] W. Wu and A.-M. S. Tremblay, Phase Diagram and Fermi Liquid Properties of the Extended Hubbard Model on the Honeycomb Lattice, Phys. Rev. B 89, 205128 (2014).

[37] L. Balents, Spin Liquids in Frustrated Magnets, Nature (London) 464, 199 (2010).

[38] Strictly speaking, a Mott insulator is defined as an insulator induced by electron correlations which occurs without breaking any conventional symmetry. However, it is generally accepted that an interaction-driven insulator is simply referred to as a Mott insulator to emphasize the importance of electron correlations [2]. Hence, we adopt this convention throughout this paper. In particular, the insulating state in both models studied here is always concomitant with the AF order, and thus it can be better referred to, in a loose sense, as an "AF Mott insulator." In this respect, it should also be noted that the insulating state studied here does not break the translation symmetry because the unit cell contains two sites and an even number of electrons. In this case, the definition of a genuine Mott insulator in the strict sense is rather ambiguous and certainly more confusing.

[39] H. F. Trotter, On the Product of Semi-Groups of Operators, Proc. Am. Math. Soc. 10, 545 (1959).

[40] M. Suzuki, Generalized Trotter's Formula and Systematic Approximants of Exponential Operators and Inner Derivations with Applications to Many-Body Problems, Commun. Math. Phys. 51, 183 (1976).

[41] J. E. Hirsch, Discrete Hubbard-Stratonovich Transformation for Fermion Lattice Models, Phys. Rev. B 28, R4059 (1983).

[42] The singularity in the momentum distribution function implies a long-distance behavior of the density matrix $\left\langle c_{i s}^{\dagger} c_{j s}\right\rangle \sim Z /\left|\boldsymbol{r}_{i}-\boldsymbol{r}_{j}\right|^{2}$, the same kind of behavior implied for a single-particle Green's function $G(\boldsymbol{k}, \omega)$ with the residue $Z$ of a quasiparticle pole, obtained after a careful integration in $\boldsymbol{k}$ and $\omega$. Here, $\boldsymbol{r}_{i}$ is the location of site $i$.

[43] A. B. Migdal, The Momentum Distribution of Interacting Fermi Particles, Sov. Phys. JETP 5, 333 (1957).

[44] By fitting the quasiparticle weight $Z$ in a form given in Eq. (10), we have found that $\eta_{Z}=0.44 \pm 0.16$ for the honeycomb lattice model and $\eta_{Z}=0.59 \pm 0.17$ for the $\pi$ flux model. Noticing that $\eta_{Z}=\nu \eta_{\psi}$, these values are almost 2 times larger than the ones estimated by the data-collapse fits in Sec. IV (see also Sec. VI). This difference is mostly due to the nontrivial extrapolation procedure for the estimation of $Z: Z$ is estimated by extrapolating $Z_{L}$ in the thermodynamic limit with a polynomial function of $1 / L$, where $Z_{L}$ is first obtained by extrapolating $n\left(\varepsilon_{\boldsymbol{k}}\right)$ to the Fermi level, thus involving two extrapolations. Therefore, we rely more on the values obtained by the finite-size scaling analysis in Sec. IV, where (i) no extrapolation procedure is involved and (ii) all data points are used not only for $U<U_{\mathrm{c}}$ but also for $U>U_{\mathrm{c}}$.
[45] Finite-Size Scaling, edited by J. L. Cardy (Elsevier, Amsterdam, 1988).

[46] K. S. D. Beach, L. Wang, and A. W. Sandvik, Data Collapse in the Critical Region Using Finite-Size Scaling with Subleading Corrections, arXiv:cond-mat/0505194.

[47] M. Campostrini, A. Pelissetto, and E. Vicari, Finite-Size Scaling at Quantum Transitions, Phys. Rev. B 89, 094516 (2014).

[48] The leading correction to scaling is not generally proportional to $f_{m}\left(u L^{1 / \nu}\right)$, but it may contain different contributions [47]. Thus, our expression for the leading correction in Eq. (11) and, in particular, the value of $\omega$ should not be considered as universal but effective one to a generic correction-to-scaling exponent [46].

[49] K. Harada, Bayesian Inference in the Scaling Analysis of Critical Phenomena, Phys. Rev. E 84, 056704 (2011).

[50] The results obtained by $4-\epsilon$ expansion reported in Ref. [31] seem to be controversial since there is a discrepancy in the interpretation of the number of fermion components as, according to Herbut et al. [25,32], the number of fermion components in the chiral Heisenberg case defined in Ref. [31] has to be multiplied by 2 , in order to match the previous results by $4-\epsilon$ expansion at the one-loop level [25]. Although the second-order expansion around the upper critical dimension is believed to be the most accurate method to analytically evaluate the critical exponents, it is quite clear that, at present, this expansion has not been definitively settled for the GN model.

[51] L. Kärkkäinen, R. Lacaze, P. Lacock, and B. Petersson, Critical Behaviour of the Three-Dimensional GrossNeveu and Higgs-Yukawa Models, Nucl. Phys. B415, 781 (1994).

[52] S. Chandrasekharan and A. Li, Quantum Critical Behavior in Three Dimensional Lattice Gross-Neveu Models, Phys. Rev. D 88, 021701(R) (2013).

[53] J. A. Gracey, Three-Loop Calculations in the $\mathrm{O}(N)$ Gross-Neveu Model, Nucl. Phys. B341, 403 (1990).

[54] J. A. Gracey, Computation of Critical Exponent $\eta$ at $\mathrm{O}\left(1 / N^{3}\right)$ in the Four-Fermi Model in Arbitrary Dimensions, Int. J. Mod. Phys. A 09, 727 (1994).

[55] A. N. Vasil'ev, S. É. Derkachev, N. A. Kivel', and A. S. Stepanenko, The 1/n Expansion in the Gross-Neveu Model: Conformal Bootstrap Calculation of the Index $\eta$ in Order $1 / n^{3}$, Theor. Math. Phys. 94, 127 (1993).

[56] L. Rosa, P. Vitale, and C. Wetterich, Critical Exponents of the Gross-Neveu Model from the Effective Average Action, Phys. Rev. Lett. 86, 958 (2001).

[57] F. Höfling, C. Nowak, and C. Wetterich, Phase Transition and Critical Behavior of the $d=3$ Gross-Neveu Model, Phys. Rev. B 66, 205111 (2002).

[58] L. Wang, P. Corboz, and M. Troyer, Fermionic Quantum Critical Point of Spinless Fermions on a Honeycomb Lattice, New J. Phys. 16, 103008 (2014).

[59] Z.-X. Li, Y.-F. Jiang, and H. Yao, Fermion-Sign-Free Majorana-Quantum-Monte-Carlo Studies of Quantum Critical Phenomena of Dirac Fermions in Two Dimensions, New J. Phys. 17, 085003 (2015).

[60] S. Hesselmann and S. Wessel, Thermal Ising Transitions in the Vicinity of Two-Dimensional Quantum Critical Points, arXiv:1602.02096. 
[61] D. J. Amit, Field Theory, Renormalization Group, and Critical Phenomena (World Scientific, Singapore, 1984).

[62] V. Privman and M. E. Fisher, Universal Critical Amplitudes in Finite-Size Scaling, Phys. Rev. B 30, 322 (1984).

[63] D. Pines and P. Nozières, The Theory of Quantum Liquids (Addison-Wesley, Menlo Park, 1966).

[64] M. Polini, R. Asgari, Y. Barlas, T. Pereg-Barnea, and A. H. MacDonald, Graphene: A Pseudochiral Fermi Liquid, Solid State Commun. 143, 58 (2007).

[65] R. P. Feynman and M. Cohen, Energy Spectrum of the Excitations in Liquid Helium, Phys. Rev. 102, 1189 (1956).

[66] M. Capello, F. Becca, M. Fabrizio, S. Sorella, and E. Tosatti, Variational Description of Mott Insulators, Phys. Rev. Lett. 94, 026406 (2005).

[67] M. Capello, F. Becca, S. Yunoki, and S. Sorella, Unconventional Metal-Insulator Transition in Two Dimensions, Phys. Rev. B 73, 245116 (2006).
[68] For example, in the exact infinite-dimension calculation, which is well known to be equivalent to the Gutzwiller approximation, it can be shown that $g_{\mathrm{NN}}=(4 \bar{d})^{2}[69]$.

[69] F. Gebhard, Gutzwiller Correlated Wave Functions in Finite Dimensions d: A Systematic Expansion in 1/d, Phys. Rev. B 41, 9452 (1990).

[70] G. Kotliar, The Large N Expansion in the Strong Correlation Problem, in Les Houches Session LVI 1991, edited by B. Doucot and J. Zinn-Justin (Elsevier, Amsterdam, 1995), p. 197.

[71] S. Yunoki, E. Dagotto, and S. Sorella, Role of Strong Correlation in the Recent Angle-Resolved Photoemission Spectroscopy Experiments on Cuprate Superconductors, Phys. Rev. Lett. 94, 037001 (2005).

[72] L. Wang, Y.-H. Liu, and M. Troyer, Stochastic Series Expansion Simulation of the $t-V$ Model, arXiv:1602.02095. 